\title{
Resonance refraction and neutrino oscillations
}

\author{
Alexei Y. Smirnov ${ }^{a, c}$ and Victor B. Valera, ${ }^{b, c, 1}$ \\ ${ }^{a}$ Max-Planck-Institut für Kernphysik, \\ 69117 Heidelberg, Germany \\ ${ }^{b}$ Niels Bohr International Academy, Niels Bohr Institute, \\ University of Copenhagen, DK-2100 Copenhagen, Denmark \\ ${ }^{c}$ Abdus Salam International Centre for Theoretical Physics, \\ Strada Costiera 11, 34014 Trieste, Italy \\ E-mail: smirnov@mpi-hd.mpg.de, vvalera@nbi.ku.dk
}

ABSTRACT: The refraction index and matter potential depend on neutrino energy and this dependence has a resonance character associated to the production of the mediator in the $s$-channel. For light mediators and light particles of medium (background) the resonance can be realized at energies accessible to laboratory experiments. We study properties of the energy dependence of the potential for different C-asymmetries of background. Interplay of the background potential and the vacuum term leads to (i) bump in the oscillation probability in the resonance region, (ii) dip related to the MSW resonance in the background, (iii) substantial deviation of the effective $\Delta m^{2}$ above the resonance from the low energy value, etc. We considered generation of mixing in the background. Interactions with background shifts the energy of usual MSW resonance and produces new MSW resonances. Searches of the background effects allow us to put bounds on new interactions of neutrinos and properties of the background. We show that explanation of the MiniBooNE excess, as the bump due to resonance refraction, is excluded.

Keywords: Neutrino Physics, Beyond Standard Model

ArXiv EPrint: 2106.13829

\footnotetext{
${ }^{1}$ Corresponding author.
} 


\section{Contents}

1 Introduction 1

2 Potentials and resonances 3

2.1 Neutrino interactions with new light sector 3

2.2 Potentials in the fermionic background 4

2.3 Potentials in the bosonic background 6

2.4 Resonance, energy smearing, coherence $\quad 6$

2.5 Properties of resonance and total potential 9

3 Resonance refraction and oscillations $\quad 11$

$\begin{array}{ll}3.1 \text { Background versus vacuum contributions } & 11\end{array}$

$\begin{array}{lll}3.2 & \text { Effective mass splitting } & 13\end{array}$

$\begin{array}{llr}3.3 & \text { Negative } \kappa & 14\end{array}$

$\begin{array}{lll}3.4 & \text { Phases and probabilities } & 15\end{array}$

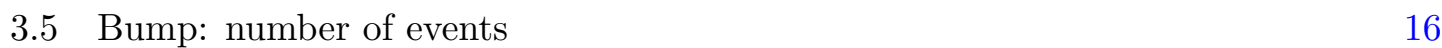

$\begin{array}{ll}3.6 & \text { Adding usual matter effect } \\ \end{array}$

$\begin{array}{lll}3.7 & \text { Generation of mixing in the background } & 19\end{array}$

$\begin{array}{lll}3.8 & \text { Resonance refraction and Glashow resonance } & 21\end{array}$

4 Applications to specific experiments 21

$\begin{array}{ll}4.1 & \text { Signatures and implications } \\ & 21\end{array}$

4.2 MiniBooNE excess and resonance refraction 22

$\begin{array}{lll}4.3 & \text { Bounds on the background effects } & 23\end{array}$

5 Conclusions $\quad 26$

\section{Introduction}

The Wolfenstein potential, ${ }^{1}$ which describes the matter effect on neutrino oscillations, does not depend on the neutrino energy [1-4]. This is the consequence of

(i) large mass of mediators of interactions, $M_{\text {med }}$, or low energies of neutrinos, so that the total energy in the CMS: $\sqrt{s} \ll M_{\text {med }}$. Recall that originally the potentials were derived using the 4 fermion point-like interactions.

(ii) the $\mathrm{C}$ - (CP-) asymmetry of background. In the C-symmetric medium in the lowest order the potentials are zero.

\footnotetext{
${ }^{1}$ In what follows we will consider potentials which are related to the refraction index $n$ as $V=(n-1) p$, where $p$ is the momentum of neutrino.
} 
In general (independently of the C-asymmetry) substantial dependence of the potentials on energy should show up at energies $\sqrt{s} \simeq M_{\text {med }}$. Furthermore, exchange of mediator in the $s$-channel leads to the resonance character of this dependence [5]. We will call this phenomenon the resonance refraction.

In the Standard Model the mediators of neutrino interactions are $W, Z^{0}$ as well as $H^{0}$. $Z^{0}$ leads to the resonance refraction in the $\bar{\nu} \nu$ - annihilation. In resonance the potential is exactly zero and changes the sign with energy change. Above the resonance energy the potential has $1 / E$ dependence similar to the usual kinetic term related to mass squared difference [5]. In principle, this refraction can be realized in scattering on the ultra high energy cosmic neutrinos on relic neutrino background ( $E \geq 10^{21} \mathrm{eV}$ in the present epoch) [5]. The $W$-boson exchange produces the resonance refraction in the $\bar{\nu}_{e} e-$ scattering, i.e., in the Glashow resonance. For electrons at rest this requires the neutrino energy $\sim 6.4 \mathrm{PeV}$. We comment on possibility of observational effects in section 3.8.

For light mediators and light scatterers (their existence implies physics beyond the SM) the resonance refraction can be realized at low energies accessible to existing experiments. The resonance refraction leads to increase of the oscillation phase which can dominate over the vacuum phase in the energy range around the resonance. This produces an enhancement of the oscillation effect which would be negligible without resonance refraction. Such an enhancement was used in [6] to explain the low energy excess of the MiniBooNE events [7]. In this explanation the medium was composed of the overdense relic neutrinos.

Potentials induced by light mediator in medium with light scatterers were computed recently in connection to possible existence of light dark sector and light dark matter [8-14]. Mediators and scatterers of different nature were explored: fermions, scalars, gauge bosons. Various bounds on couplings of neutrinos with new light sector were obtained [15-30].

In this paper we focus on phenomenon of resonance refraction itself presenting results in a model independent way. We study in detail dependence of the resonant potentials on energy for different values of the $C$ - asymmetry of background. We consider interplay of resonance potentials with usual vacuum (kinetic) term as well with usual matter potential. New interesting features are realized, such as shift of the usual MSW resonances, increase or decrease of the effective mass squared difference with energy, etc. We identify signatures of the resonance refraction and outline possible observable effects. As an illustration, we apply our results to the MiniBooNE excess and show that explanation [6] is excluded. In general, applications can include explanations of some energy localized anomalies. In the absence of anomalies the bounds can be established on background parameters (densities, characteristics of scatterers) and neutrino couplings.

The paper is organized as follows. In section 2 we introduce interactions of neutrinos with new light sector. We compute potentials due these interactions and study resonances in these potentials. In section 3 we discuss effects of interplay of the background potential with vacuum (kinetic) term and usual matter potential. We consider possible observational effects and applications of the results, in particular, to an explanation of the MiniBooNE excess in section 4 . Conclusions follow in section 5. 


\section{Potentials and resonances}

\subsection{Neutrino interactions with new light sector}

In this paper we focus on phenomenon of resonance refraction itself, and present our results in general and universal form valid for different mediators and particles of background. We consider the simplest (minimal) light sector composed of new scalar $\phi$ (which can be real or complex) with mass $m_{\phi}$ and fermion $\chi$ with mass $m_{\chi}$. We comment on some extensions of this sector later. Interactions of the SM neutrino mass states $\nu_{i L}(i=1,2,3)$ with these new particles are described by

$$
\mathcal{L}^{N S I} \supset g_{i} \bar{\chi} \nu_{i L} \phi^{*}+\text { h.c. }
$$

$\phi$ may acquire VEV, thus contributing to neutrino mass. Then for single $\chi$ only one neutrino (combination of $\nu_{i}$ ) will acquire mass by VEV of $\phi$. We assume that some other sources of $\chi$ and neutrino masses exist, e.g. the see-saw mechanism, so that $\chi$ and all $\nu_{i}$ acquire different masses and in general these masses are not related to $g_{i}$.

As an option several new fermions $\chi_{j}$ can be introduced. Notice that $\chi_{i}$ themselves can be 4 component Dirac particles which implies more degrees of freedom. $\chi_{R}$ can be the left antineutrino, so that neutrinos are Majorana particles.

The coupling can be generated via mixing of singlet scalar field $\phi$ with the Higgs boson doublet (Higgs portal) [31]. Alternatively, $\phi$ can couple with RH singlet (sterile) neutrino, which in turn, couples (mixes) with active neutrinos (lepton and Higgs doublets) - that is, via the RH neutrino portal. In the Majorana case the singlet $\phi$ should mix with the neutral component of the Higgs triplet.

The couplings (2.1) were considered in various contexts before [16, 19-21, 23, 24, 27, 29]. For light new particles $m_{\phi}, m_{\chi} \ll 1 \mathrm{GeV}$, a number of generic bounds were obtained. The bounds are based on possible transitions $\nu \rightarrow \chi+\phi$.

Notice that refraction is induced by the elastic forward scattering being proportional to $g^{2} / M_{\text {med }}^{2}$. Therefore it does not disappear in the limit $g \rightarrow 0$, provided that $M_{\text {med }}$ decreases in the same way as $g$. This allows us to avoid most of the bounds based on the inelastic processes for which $\sigma \propto\left(g^{2} / q^{2}\right)^{2}$, and the transfer momentum squared $q^{2}$ is restricted from below by condition of observability.

The laboratory bounds on $g$ are rather weak: $g_{\phi} \lesssim 10^{-3}$ for masses $m_{\phi}<m_{K}(K-$ meson mass). They follow, in particular, from additional contribution to the decay $K \rightarrow$ $\mu \chi \phi$. Much stronger bounds follow from Cosmology (BBN, CMB data, structure formation) and astrophysics (star cooling, supernova dynamics and SN87A neutrino observations). They give the bound

$$
g_{\phi} \lesssim 10^{-7} .
$$

Elastic forward scattering due to the interactions (2.1) produces the effective potentials $V_{i}$ for neutrino mass states in medium. There are two possibilities even for simplest case of (2.1) (i) $\phi$ plays the role of mediator while $\chi$ form a background, and vice versa: (ii) $\chi$ is the mediator while $\phi$ form a background. 


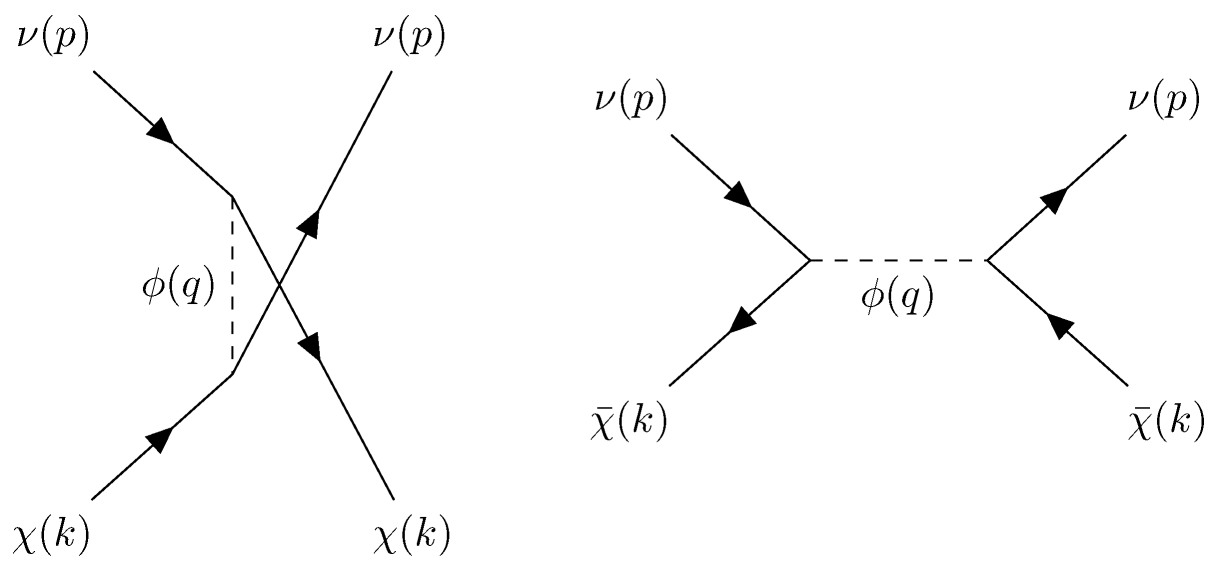

Figure 1. Feynman diagrams for scattering of neutrinos on a background composed of fermions $\chi$ (left) and antifermions $\bar{\chi}$ (right).

\subsection{Potentials in the fermionic background}

We consider first the case of strong hierarchy of couplings: $g_{3} \gg g_{2}, g_{1}$, so that $\nu_{3}$ couples with background, while interactions of others can be neglected. In this case the interactions, and consequently the potentials, are diagonal in the mass basis. We will discuss couplings of all three neutrinos later in section 3.7. Also we comment on the case of three $\chi_{j}$.

We consider background composed of fermions $\chi$ and antifermions $\bar{\chi}$ with number densities $n_{\chi}$ and $\bar{n}_{\chi}$ correspondingly. The C-asymmetry of the background can be defined as

$$
\epsilon \equiv \frac{n_{\chi}-\bar{n}_{\chi}}{n_{\chi}+\bar{n}_{\chi}}
$$

The mediator is a scalar $\phi$ and the diagrams of the neutrino scattering on $\chi$ (left) and $\bar{\chi}$ (right) are shown in figure 1. For $m_{\phi}>m_{\nu}, m_{\chi}$ the right diagram with the s-channel exchange produces resonance.

To obtain the potential, we integrate the matrix element of the process over the momentum of particle $\chi$ with distribution function, $F_{\chi}(k)$. The latter is normalized as

$$
\int d^{3} k F_{\chi}(k)=n_{\chi},
$$

and similarly for $\bar{\chi}$. The left ( $u$-channel) and right ( $s$-channel) diagrams in figure 1 give correspondingly the potentials

$$
\begin{aligned}
V_{u i} & =\int d^{3} k F_{\chi}(k)\left\langle\nu_{i, p} \chi_{k}\left|g_{i}^{\dagger} \bar{\nu}_{i} P_{R} \chi \frac{1}{q^{2}-m_{\phi}^{2}} g_{i} \bar{\chi} P_{L} \nu_{i}\right| \nu_{i, p} \chi_{k}\right\rangle, \\
V_{s i} & =\int d^{3} k F_{\bar{\chi}}(k)\left\langle\nu_{i, p} \bar{\chi}_{k}\left|g_{i}^{\dagger} \bar{\nu}_{i} P_{R} \chi \frac{1}{q^{2}-m_{\phi}^{2}+i m_{\phi} \Gamma_{\phi}} g_{i} \bar{\chi} P_{L} \nu_{i}\right| \nu_{i, p} \bar{\chi}_{k}\right\rangle,
\end{aligned}
$$

Here in the propagator we added the term with total width of $\phi$. In vacuum

$$
\Gamma_{\phi}^{0}=\sum_{i} \frac{g_{i}^{2}}{8 \pi} m_{\phi} \approx \frac{g^{2}}{8 \pi} m_{\phi},
$$


$(\phi \rightarrow \nu \chi)$, where $g=g_{3}$ and we take $g_{1}=g_{2}=0$. In medium the term with $\Gamma$ is modified (see below).

We assume first that the background particles are at rest which is valid for cold gases like dark matter $(\mathrm{DM})$ or relic neutrinos from the cosmological neutrino background $(\mathrm{C} \nu \mathrm{B})$. Then $F_{\chi}(k)=n \delta(\vec{k})$ and the integrals in (2.5) and (2.6) give the total potential

$$
V^{B} \equiv V_{u}+V_{s}=\frac{|g|^{2}}{2}\left[\frac{n_{\chi}}{\left(2 E_{\nu} m_{\chi}+m_{\phi}^{2}\right)}+\frac{\bar{n}_{\chi}\left(2 E_{\nu} m_{\chi}-m_{\phi}^{2}\right)}{\left(2 E_{\nu} m_{\chi}-m_{\phi}^{2}\right)^{2}+\left(m_{\phi} \Gamma_{\phi}\right)^{2}}\right] .
$$

This expression differs from expression for potential in [6], but coincides with that in [11].

We obtain similar result for moving $\chi$ with the only substitution $m_{\chi} \rightarrow E_{\chi}$, if the angular distribution is isotropic. This is important for the degenerate gas with large overdensity when the Fermi momentum $p_{f} \gg m_{\chi}$.

The second term in (2.8) has a resonance dependence on energy (pole of propagator) with the resonance energy

$$
E_{R} \equiv \frac{m_{\phi}^{2}-m_{\chi}^{2}-m_{\nu}^{2}}{2 m_{\chi}} \approx \frac{m_{\phi}^{2}}{2 m_{\chi}} .
$$

At $E_{R}$ the contribution $V_{s}$ is exactly zero and it changes the sign with energy change. The amplitude of scattering becomes purely imaginary, which corresponds to production of the on shell $\phi$. In terms of the resonance energy the potential (2.8) can be rewritten as

$$
V^{B}=\frac{|g|^{2}\left(n_{\chi}+\bar{n}_{\chi}\right)}{8 m_{\chi}}\left[\frac{\left(E-E_{R}\right)(1-\epsilon)}{\left(E-E_{R}\right)^{2}+\left(\xi E_{R}\right)^{2}}+\frac{1+\epsilon}{E+E_{R}}\right],
$$

where

$$
\xi \equiv \frac{\Gamma_{\phi}}{m_{\phi}}
$$

and in vacuum

$$
\xi^{0} \equiv \frac{g^{2}}{8 \pi}
$$

Let us introduce a dimensionless parameter

$$
y \equiv \frac{E}{E_{R}} .
$$

In terms of $y$ the expression for the potential (2.10) becomes

$$
V^{B}=\frac{1}{2} V_{0}^{B}\left[\frac{(1-\epsilon)(y-1)}{(y-1)^{2}+\xi^{2}}+\frac{1+\epsilon}{y+1}\right]
$$

where

$$
V_{0} \equiv \frac{g^{2}}{2 m_{\phi}^{2}}\left(n_{\chi}+\bar{n}_{\chi}\right)
$$

In this way we can disentangle dependencies of the potential on relevant physical quantities: $V_{0}$ depends on parameters of mediator, $g$ and $m_{\phi}$, and on total density of scatterers in a background. It has a form of the standard matter potential at low energies with $G_{\phi}=$ $g^{2} / 2 m_{\phi}^{2}$. The parameter $\xi$ is proportional to the coupling constant squared, while the mass of $\chi$ enters via $E_{R}$. $V_{0}$ is introduced in such a way that for $y \rightarrow 0$ we have $V^{B} \rightarrow \epsilon V_{0}$, and consequently, for $\epsilon= \pm 1: V^{B}= \pm V_{0}$, thus reproducing the standard Wolfenstein potential. 


\subsection{Potentials in the bosonic background}

For the scalar particle background and fermionic mediator the potential is similar to the one computed before. In the lowest order in $g^{2}$, up to factor of 2 the potential has the same expression as in (2.8) with the following substitutions

$$
m_{\phi} \leftrightarrow m_{\chi}, \quad n_{\chi} \rightarrow n_{\phi}, \quad \Gamma_{\phi} \rightarrow \Gamma_{\chi}
$$

Thus,

$$
V^{\phi} \approx 2 V^{\chi}\left(m_{\phi} \rightarrow m_{\chi}, m_{\chi} \rightarrow m_{\phi}, n_{\chi} \rightarrow n_{\phi}, \Gamma_{\phi} \rightarrow \Gamma_{\chi}\right)
$$

The resonance is realized if $m_{\chi}>m_{\nu}+m_{\phi}$, and the resonance energy equals

$$
E_{R} \simeq \frac{m_{\chi}^{2}}{2 m_{\phi}}
$$

In terms of resonance energy the potential can be written in exactly the same form as in (2.14) with

$$
V_{0}^{\phi}=\frac{g^{2}}{2 m_{\chi}^{2}}\left(n_{\phi}+\bar{n}_{\phi}\right)
$$

and

$$
\epsilon^{\phi} \equiv \frac{n_{\phi}-\bar{n}_{\phi}}{n_{\phi}+\bar{n}_{\phi}}
$$

The difference from the fermionic background case may appear in higher orders in $g^{2}$ due to fermionic nature of mediator $\chi$. Now the amplitude of scattering is proportional to $\not q=\not p+\not k: A=\not p \Sigma_{\nu}+\not k \Sigma_{\chi}$. The first term gives contribution to renormalization of the wave function of neutrino: $\nu=\left(1+\Sigma_{\nu} / 2\right) \nu_{L}^{\prime}$, while the second one generates the potential: for the background at rest $\gamma^{0} m_{\phi} \Sigma_{\chi}=\gamma^{0} V$. Renormalization leads to change of the potential: $V^{\prime}=\left(1+\Sigma_{\nu}^{*} / 2\right) V\left(1+\Sigma_{\nu} / 2\right)=V\left(1+\Sigma_{\nu}\right)$ (as well as usual kinetic term) [11]. The correction is of the order $g^{2}$. In this order one should take into account also loop corrections to external neutrino lines All these corrections have the same nature and can be described by tree level diagrams with multiple scattering on a background: $\nu+\phi^{*} \rightarrow \chi \rightarrow \nu+\phi^{*}, \nu+\phi^{*} \rightarrow \chi \ldots$. Alternatively it can be treated as resummation of self-energy loop diagrams. The high order corrections will not change general properties (energy dependence) of potentials. In the lowest order properties of the resonances in the scalar and fermion backgrounds are the same. The difference appears in applications and implications for theory.

\subsection{Resonance, energy smearing, coherence}

In resonance, $y=1$, the $s$-component of the potential (2.14) is zero for any asymmetry, $V_{s}=0$, and only non-resonance component contributes. The potential has extrema at $y=1 \pm \xi$ :

$$
\left|V^{\max }\right|=\frac{V_{0}}{4}\left(\frac{1-\epsilon}{\xi}+1+\epsilon\right) \approx \frac{V_{0}}{4} \frac{1-\epsilon}{\xi} .
$$


So, in resonance the enhancement is given by inverse coupling constant squared. The energy interval between two extrema equals $2 \xi E_{R}$. In these points the ratio of the resonant to non-resonant part equals

$$
\frac{V_{s}}{V_{u}}=\frac{1}{\xi} \frac{1-\epsilon}{1+\epsilon} .
$$

Zero of the total potential is shifted with respect to $y=1$ due to the non-resonant contribution as

$$
y_{0}=1-\frac{1}{2} \xi^{2} \frac{1+\epsilon}{1-\epsilon} .
$$

The width of the peak at the half of height, $V_{s}\left(y_{1 / 2}\right)=0.5 V_{s}^{\max }$, equals

$$
\left|y_{1 / 2}-1\right|=(2+\sqrt{3}) \xi \approx 3.73 \xi
$$

For values of couplings $(2.2), \xi \sim \xi^{0}<10^{-15}$, the characteristics of resonance in (2.20)(2.22) (width and enhancement in the peak) have no physical sense. One should take into account (i) smearing of the peaks due to integration with distribution of the background $\chi$ over momenta, which differ from $\delta$ function, (ii) averaging over uncertainty in neutrino energy, (iii) effect of density correction to the width of $\phi$, (iv) dumping due to resonance absorption.

Let $\sigma_{y}$ be the scale of smearing in variable $y$. The smearing leads to decrease of heights of the peaks and their widening. If $\sigma_{y} \gg \xi$, we can neglect $\xi^{2}$ in (2.14). Then the height of the peak after averaging can be estimated as

$$
\left|V^{\max }\right|=V^{B}\left(1+\sigma_{y}\right)=V_{0}(1-\epsilon) \frac{1}{2 \sigma_{y}} .
$$

So that the enhancement factor is given by $1 / \sigma_{y}$. The maxima shift to $y \approx \sigma_{y} / 2$. Let us consider possible origings of $\sigma_{y}$.

The quantity $\sigma_{y}$ can be the width of $F_{\chi}(k)$ distribution. Recall that deriving the potential (2.10) we assumed that the background particles are at rest, $k_{\chi}=0$. (This can be still a possibility for condensate of scalar DM). For fermions $F(k)$ is not the $\delta$-function, but distribution with finite width. In eq. (2.8) one should use (even for isotropic background) $E_{\chi}=\sqrt{m_{\chi}^{2}+k_{\chi}^{2}}$. Near the resonance

$$
\sigma_{y} \approx \frac{\Delta E_{\chi}}{E_{\chi}}
$$

and for non-relativistic background $E_{\chi} \sim m_{\chi}+k^{2} / 2 m_{\chi}$, so that $\Delta E_{\chi} \approx \Delta\left(k^{2}\right) / 2 m_{\chi}$. For thermal background with temperature $T$ we can take $\Delta k^{2}=(3 T)^{2}$, and therefore

$$
\sigma_{y}=\frac{\Delta\left(k^{2}\right)}{2 m_{\chi}^{2}} \approx \frac{9 T^{2}}{2 m_{\chi}^{2}} .
$$

If $T=1.945 \mathrm{~K}$ and $m_{\chi}=0.05 \mathrm{eV}$, we obtain the value of enhancement $1 / \sigma_{y} \sim 10^{4}$.

Further smearing of the dependence of potential on energy is due to neutrino energy uncertainty $\sigma_{E}$ in the oscillation setup. In this case $\sigma_{y}=\sigma_{E} / E_{R}$. 
For very narrow resonance one needs to take into account the medium corrections to the $\phi$-propagator. The main correction is given by the loop diagram $\phi \rightarrow \nu+\chi^{*} \rightarrow \phi$ with the $\chi$ propagator in a finite density medium. This medium correction corresponds to scattering of $\phi$ on particles of medium via neutrino as mediator: $\phi+\chi \rightarrow \nu \rightarrow \phi+\chi$. So, whole the process consists of the transitions: $\nu+\chi^{*} \rightarrow \phi, \phi+\chi \rightarrow \nu, \nu+\chi^{*} \rightarrow \phi$, $\phi \rightarrow \chi+\nu$. These transitions can be treated as the induced decay of $\phi$ in medium. The polarization operator equals

$$
\Pi=g^{2} \frac{n_{\chi}}{4 m_{\chi}},
$$

which should be compared with $m_{\phi} \Gamma_{\phi}^{0}=g^{2} m_{\phi}^{2} / 8 \pi$. Therefore the width can be written as

$$
\Gamma_{\phi}=\Gamma_{\phi}^{0}\left(1+\frac{2 \pi n_{\chi}}{m_{\phi}^{2} m_{\chi}}\right) \text {. }
$$

Ratio of the polarization operator and $m_{\phi}^{2}$ (the denominator outside the resonance):

$$
\beta \equiv \frac{\Pi}{m_{\phi}^{2}}=\frac{g^{2} n_{\chi}}{4 m_{\phi}^{2} m_{\chi}} .
$$

can be considered as the expansion parameter of the perturbation theory.

Refraction implies coherence: zero transfer momentum by neutrinos, and consequently, the unchanged state of medium $|M\rangle:\left\langle M^{\prime} \mid M\right\rangle \approx 1$. In the resonance region (in the schannel) $\nu$ interacting with $\chi$ in some point $x$ produces nearly on-shell $\phi$ which propagates for some distance and then decays back into $\nu$ and $\chi$. So, the particle of medium reappears in different space-time point $x^{\prime}$. Then the coherence condition requires $\left\langle\chi\left(x^{\prime}\right) \mid \chi(x)\right\rangle \approx$ 1. That is, the corresponding wave functions of $\chi$ before and after scattering should nearly coincide.

The time of propagation of $\phi$ between the production and annihilation is determined by the decay rate $\tau_{\phi}=1 / \Gamma$. Taking into account the Lorentz factor $\gamma=E_{\phi} / m_{\phi}$ we find the distance of propagation of $\phi$ in the rest frame of background:

$$
d \approx c t_{\phi}=\tau_{\phi} \frac{E_{\phi}}{m_{\phi}}=\frac{2 \pi E_{\phi}}{|g|^{2} m_{\phi}^{2}} .
$$

For light background particles the total energy of mediator is $E_{\phi}=E+m_{\chi} \approx E_{\nu}$, Using the resonance condition, $m_{\phi}^{2}=2 E m_{\chi}$, we can rewrite $(2.27)$ as

$$
d=\frac{\pi}{|g|^{2} m_{\chi}} \approx 6.2 \cdot 10^{9} \mathrm{~cm}\left(\frac{|g|}{10^{-7}}\right)^{-2}\left(\frac{m_{\chi}}{1 \mathrm{eV}}\right)^{-1}
$$

$d$ should be smaller than the uncertainty in the position (localization) of the background particle $\Delta x \approx 1 / \Delta p_{\chi}$. This gives the coherence condition

$$
d<\frac{1}{\Delta p_{\chi}},
$$

which imposes the upper bound on the uncertainty

$$
\Delta p_{\chi} \lesssim 3\left(\frac{|g|}{10^{-7}}\right)^{2}\left(\frac{m_{\chi}}{1 \mathrm{eV}}\right) 10^{-15} \mathrm{eV} .
$$


However, for a given neutrino energy most of the particles of a background are not in resonance exactly and produced $\phi$ will be out of mass shell. The virtuality can be estimated as

$$
\Delta q \sim \sqrt{s-m_{\phi}^{2}}=\sqrt{E_{R} \frac{k^{2}}{m_{\chi}}}=\frac{m_{\phi} k}{m_{\chi}} .
$$

Consequently, typical distance of travel is $d \sim m_{\chi} / m_{\phi} k$. The scale of localization is about $1 / n_{\chi}^{1 / 3}$. So, the condition for coherence can be written as

$$
\frac{m_{\chi}}{m_{\phi}\langle k\rangle} \ll \frac{1}{n_{\chi}^{1 / 3}} .
$$

\subsection{Properties of resonance and total potential}

Outside the resonance, $|y-1| \gg \xi$, neglecting $\xi$ we obtain from (2.14)

$$
V^{B}(y, \epsilon)=V_{0} \frac{y-\epsilon}{y^{2}-1} .
$$

In figure 2 we show dependence of $V^{B} / V_{0}$ on $y$ for different values of asymmetry $\epsilon(\epsilon=$ $-1 \div 1)$. For $y=0$ :

$$
V^{B}=V_{0} \epsilon,
$$

so that for symmetric background $V^{B}=0$. Above the resonance, $y \gg 1$, independently of $\epsilon$

$$
V \approx V_{0} \frac{1}{y} \text {. }
$$

Thus, at $E \gg E_{R}$ the potential takes the form of the standard vacuum contribution with $1 / E$ dependence. Therefore, in principle, the standard neutrino oscillations can be reproduced (even for massless neutrinos) provided that

$$
\frac{n_{\chi}}{4 m_{\chi}} \Delta|g|^{2} \simeq \Delta m^{2} .
$$

(See recent discussion in $[13,32])$.

For particular values of $\epsilon$ we have the following dependence on $y$ (see figure 2).

- $\epsilon=-1$ corresponds to pure $\bar{\chi}$ background, and consequently, only the resonance contribution exists:

$$
V^{B}(y,-1)=V_{0} \frac{1}{y-1} .
$$

At $y=0: V(0,-1)=-V_{0}$, then it decreases with increase of $y$.

With increase of $\epsilon$ the low energy part of the potential $(y<1)$ shifts up.

- $\epsilon=0$ corresponds to symmetric background. The potential equals

$$
V^{B}(y, 0)=V_{0} \frac{y}{y^{2}-1} .
$$

$V=0$ at $y=0$, and then $V^{B}(y, 0)$ decreases linearly below the resonance:

$$
V(y, 0)=-V_{0} y
$$




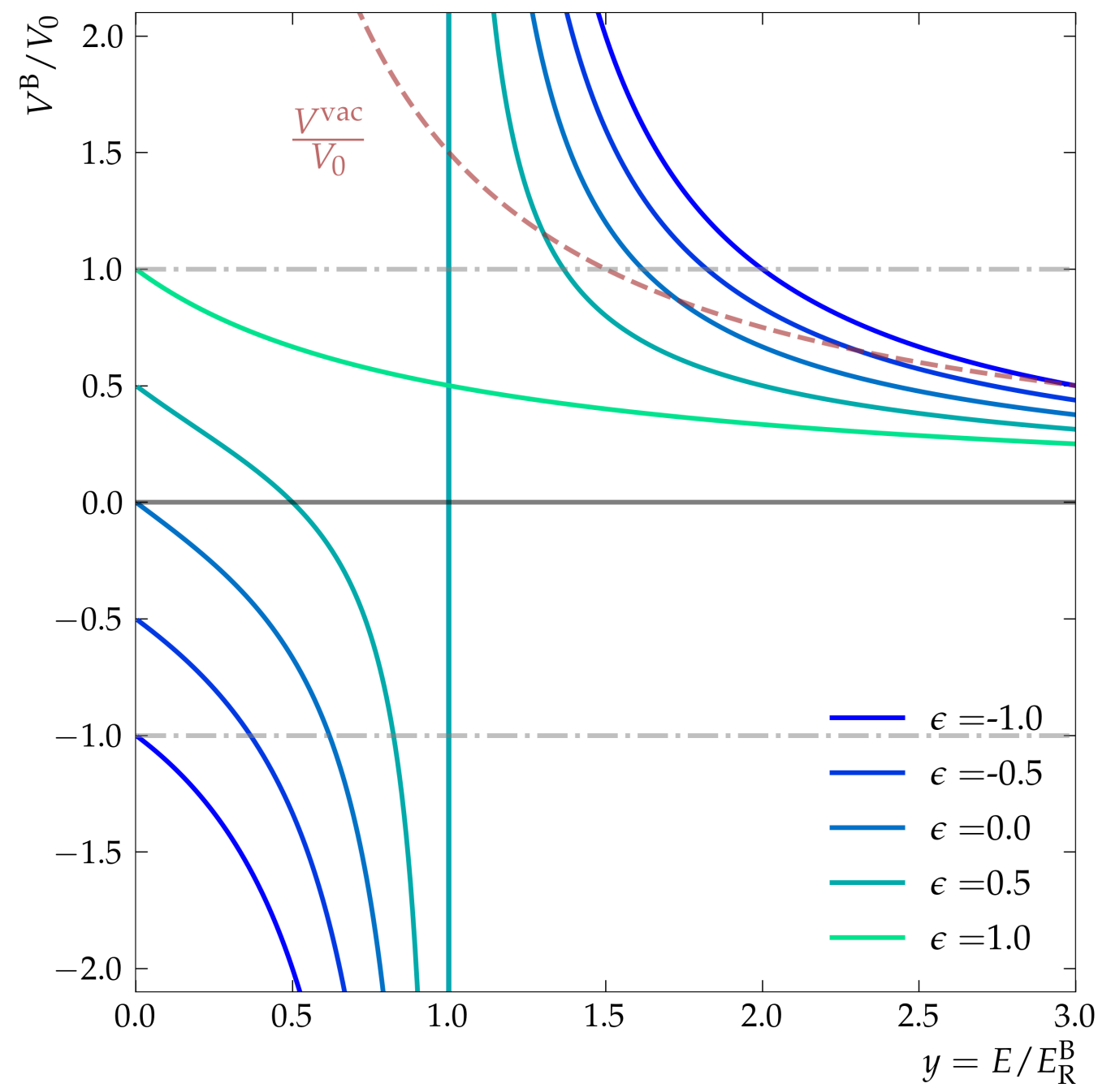

Figure 2. The dependence of the potential $V^{B} / V^{0}$ on energy, $y=E / E_{R}$, for different values of $\epsilon$. Shown also the vacuum kinetic energy $V^{\text {vac }} / V_{0}$ as function of $y$.

- $\epsilon>0$ : according to (2.32) for $y>\epsilon, V^{B}$ has positive values, it vanishes at $y=\epsilon$ and then becomes negative.

- $\epsilon=1$ corresponds to pure $\chi$ background and resonance is absent:

$$
V^{B}(y, 1)=V_{0} \frac{1}{y+1}
$$

describes the asymptotic curve with $V^{B} / V_{0}=1$ at $y=0 . V^{B}(y) / V_{0}$ decreases monotonously from 1 to 0 at $y \rightarrow \infty$ and at $y=1$ the ratio equals 0.5 .

For $\epsilon<1$ the dependence of potential on $y$ has two branches. In the low energy branch, $y<1$, the ratio $V^{B} / V_{0}$ decreases from $\epsilon$ at $y=0$ down to $-(1-\epsilon) / 4 \xi$ at $y \approx 1-\xi$, if there is no smearing, see eq. (2.20). In the high energy branch, $y>1$, we have $V^{B} / V_{0}>0$, and it decreases from $V^{B} / V_{0} \sim(1-\epsilon) / 4 \xi$ at $y=1+\xi$ down to zero at $y \rightarrow \infty$ (without 
smearing). The two branches are connected in the range $y=1 \pm \xi$. The largest effect of a background is for $\epsilon=-1$. With increase of $\epsilon$ both branches approach the non-resonance curve (2.36) everywhere apart from the region around 1 :

$$
y=\epsilon \div\left[\frac{1}{2}+\frac{1}{2} \sqrt{1+4(1-\epsilon)}\right] \approx \epsilon \div(2-\epsilon) .
$$

\section{Resonance refraction and oscillations}

\subsection{Background versus vacuum contributions}

Let us consider an interplay of the background $V^{B}$ with kinetic term ("vacuum potential"):

$$
V^{\mathrm{vac}}(E) \equiv \frac{\Delta m^{2}}{2 E}=\frac{V_{R}^{\mathrm{vac}}}{y}, \quad V_{R}^{\mathrm{vac}} \equiv \frac{\Delta m^{2}}{2 E_{R}} .
$$

We can neglect the usual matter effect if the refraction resonance energy is much smaller than the MSW resonance energy: $E_{R}^{B} \ll E_{R}^{\mathrm{MSW}}$. For the Earth based experiments this means $E_{R}^{B} \ll 6 \mathrm{GeV}$, which is realized for short baseline experiments, such as reactor neutrino experiments, LSND and MiniBooNE and low energy LBL experiments, e.g., T2K.

In general, $V^{\text {vac }}$ can be positive or negative depending on the mass ordering (sign of $\left.\Delta m^{2}\right)$. The sign is relevant since now we have two contribution to the phase. In the model where $y_{i}$ correlate with masses, the potentials $V^{\text {vac }}$ and $V_{0}$ correlate too, having the same sign. If both $V_{0}$ and $V^{\text {vac }}$ are positive, the potential $V^{\text {vac }}(y) \operatorname{crosses} V^{B}(y)$ at $y>1$ provided that $V_{R}^{\mathrm{vac}} / V_{0}>1 / 2$.

To compare the two contributions we consider the ratio

$$
\kappa(y) \equiv \frac{V^{B}}{V^{\mathrm{vac}}}=r \frac{y(y-\epsilon)}{y^{2}-1},
$$

where according to $(2.9),(2.15)$,

$$
r \equiv \frac{V_{0}}{V_{R}^{\mathrm{vac}}}=\frac{g^{2}\left(n_{\chi}+\bar{n}_{\chi}\right)}{2 m_{\chi} \Delta m^{2}} .
$$

The parameter $r$ determines the relative strength of the background effect. Notice that $r$ depends on the mass of particles of the background, but does not depend on the mass of mediator. More importantly, $r$ determines the ratio of potentials for $y \rightarrow \infty$.

Two contributions to the phase are equal (for $r \neq 1$ ) at

$$
y_{\mathrm{eq}}=\frac{1}{2(1-r)}\left[-\epsilon r+\sqrt{\epsilon^{2} r^{2}+4(1-r)}\right] .
$$

This equation gives $y_{\mathrm{eq}}=1 /(1-r)$ for $\epsilon=-1$, and $y_{\mathrm{eq}}=\sqrt{1 /(1-r)}$ for $\epsilon=0$. With decrease of $r$, as well as increase of $\epsilon$ the value of $y_{\text {eq }}$ approaches 1. For the non-resonance case $(\epsilon=1) y_{\text {eq }}=1 /(r-1)$ and the equality is realized when $r>2$.

For the low energy branch, $y<1$, an interesting feature is cancellation of two contributions when

$$
V^{B}=-V^{\mathrm{vac}},
$$




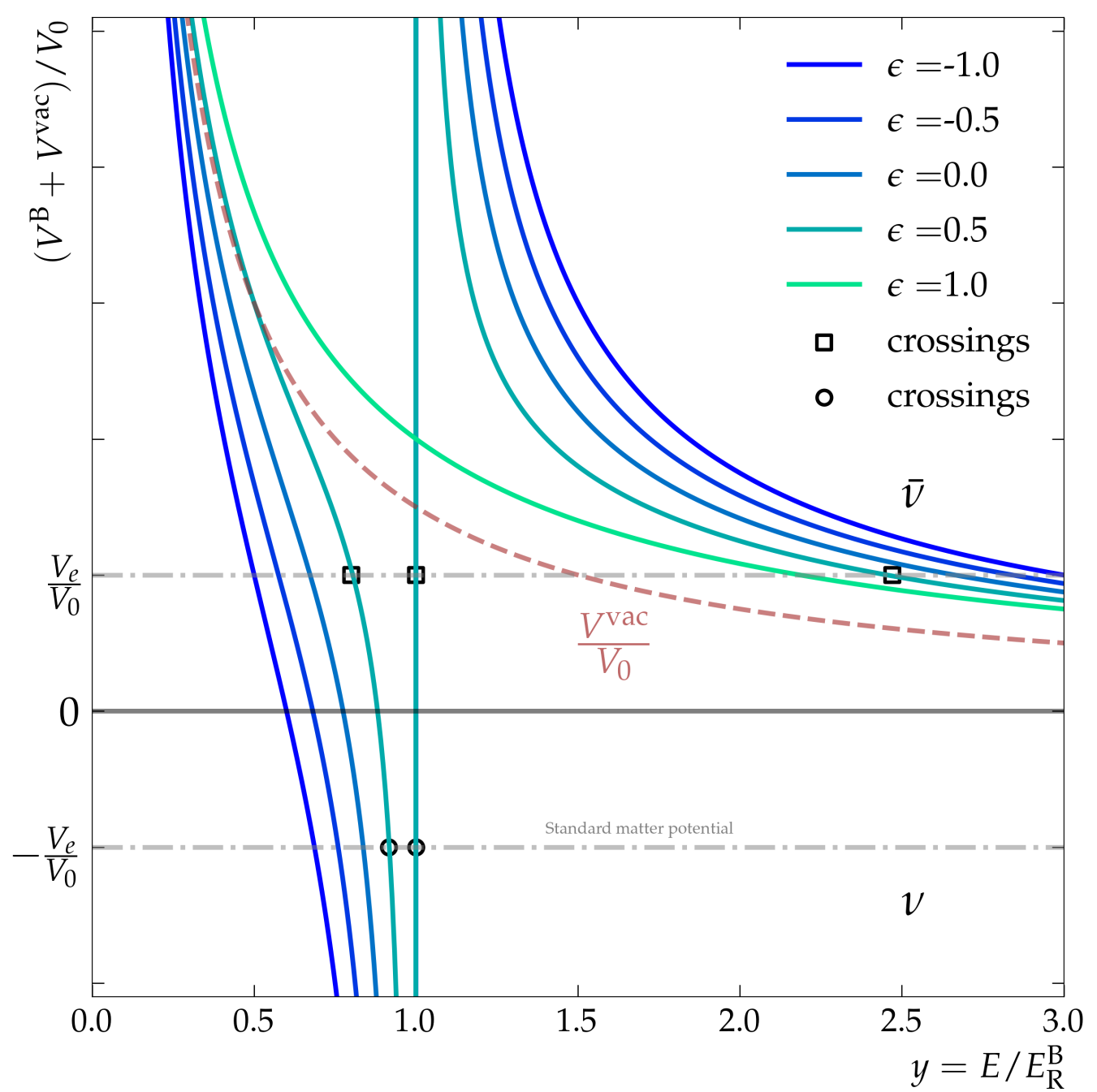

Figure 3. The dependence of the total potential, $\left(V^{B}+V^{\text {vac }}\right) / V_{0}$, on energy $y$ for different values of $\epsilon$. The horizontal lines correspond to the usual matter potential $V_{e} / V_{0}$ for neutrinos and antineutrinos. Crossings of these lines with $\left(V^{B}+V^{\mathrm{vac}}\right) / V_{0}$ show the points of the MSW resonances in the neutrino (empty boxes) and antineutrino (empty circles) channels.

which corresponds to the MSW resonance on the background. If $r \neq-1$ this happens at

$$
y_{c}=\frac{1}{2(1+r)}\left[\epsilon r+\sqrt{\epsilon^{2} r^{2}+4(1+r)}\right],
$$

so that $y_{c}=1 /(1+r)$ for $\epsilon=-1$, and $y_{c}=\sqrt{1 /(1+r)}$ for $\epsilon=0$. With decrease of $r$ and $\epsilon \rightarrow 1$ the cancellation point approaches 1 . Also for $\epsilon \rightarrow 1$ we find that $y_{c} \rightarrow 1$.

The sum of two contributions

$$
V^{\mathrm{sum}} \equiv V^{\mathrm{vac}}+V^{B}=V^{\mathrm{vac}}[1+\kappa(y)],
$$

in the units of $V_{0}$ as function of $y$, is shown in figure 3. It has the following features. In the high energy branch $V^{\text {sum }}$ increases from $\left[V^{\text {vac }}(1+r)\right]$ at $y \rightarrow \infty$ to $V_{0}(1-\epsilon) / 4 \xi$ at $y=1+\xi$ 
(in absence of smearing). The two contributions become equal at $y_{\text {eq }}$ (3.4). In the low energy branch $V^{\text {sum }} / V_{0}$ decreases from $V^{\text {vac }} / V_{0}(1+\epsilon)$ at $y \rightarrow 0$, down to $-(1-\epsilon) / 4 \xi$ at $y=1-\epsilon$. It crosses zero at $y=y_{c}$. Correspondingly, the modulus $\left|V^{\text {sum }}\right|$ increases with $y$ at $y>y_{c}$ up to $V_{0} / \xi$.

Thus, the background contribution distorts substantially the potential (and consequently, the vacuum phase) dependence on $y$ in the resonance region $y \sim 1: y_{c} \div y_{\mathrm{eq}}$. This region shrinks with increase of $r$ and $\epsilon$. Maximal distortion effect is at $\epsilon=-1$.

\subsection{Effective mass splitting}

Effect of the background can be treated as modification of the mass squared difference which depends on neutrino energy:

$$
\Delta m_{\mathrm{eff}}^{2}(y)=\Delta m^{2}[1+\kappa(y)],
$$

so, that $V^{\text {sum }}=\Delta m_{\text {eff }}^{2}(y) / 2 E$. The ratio of the effective splitting in a background, $\Delta m_{\text {eff }}^{2}$, and in vacuum, $\Delta m^{2}$ equals

$$
R_{\Delta} \equiv \frac{\Delta m_{\mathrm{eff}}^{2}}{\Delta m^{2}}=\frac{V^{\mathrm{sum}}}{V^{\mathrm{vac}}}=\frac{\Phi^{\mathrm{tot}}}{\Phi^{\mathrm{vac}}} .
$$

According to (3.7) the ratio can be written as

$$
R_{\Delta}(y)=1+r \frac{y(y-\epsilon)}{y^{2}-1} .
$$

For $y \rightarrow 0$ the correction disappears

$$
R_{\Delta}(y)=1+\epsilon r y .
$$

For high energies with increase of $y$ the ratio converges to constant value

$$
R_{\Delta}(y)=1+r
$$

independently of $\epsilon$. Thus, the key consequence of interaction with background is that $\Delta m^{2}$ extracted from data above the refraction resonance differs from $\Delta m^{2}$ extracted from low energy data.

In figure 4 we show dependence of the ratio (3.9) on $y$ for different values of $r$. Here the important point is $y_{s}$ in which corrections to the modulus of effective mass squared difference changes the sign. It is determined by

$$
\left|R_{\Delta}\right|=1,
$$

or according to (3.7) by $V^{B}(y) / V^{\mathrm{vac}}(y)=-2$. Solution of the corresponding equation gives

$$
y_{s}=\frac{1}{2(2+r)}\left[\epsilon r+\sqrt{\epsilon^{2} r^{2}+8 r(2+r)}\right] \text {. }
$$

For $\epsilon=0$, we find $y_{s}=\sqrt{2 /(2+r)}$. Consequently, for $r=1.5$ it equals $y_{s}=0.87$, and for $\epsilon=-1: y_{s}=0.75$. In the interval $y=0 \div y_{s}$ the background diminishes splitting: $\Delta m_{\text {eff }}^{2}<\Delta m^{2}$, and consequently, the oscillation phase. For $y>y_{s}: \Delta m_{\text {eff }}^{2}>\Delta m^{2}$ and the phase increases. With decrease of $r$ the correction decreases and the benchmark energies $y_{c}, y_{s}$ and $y_{\text {eq }}$ approach 1. 


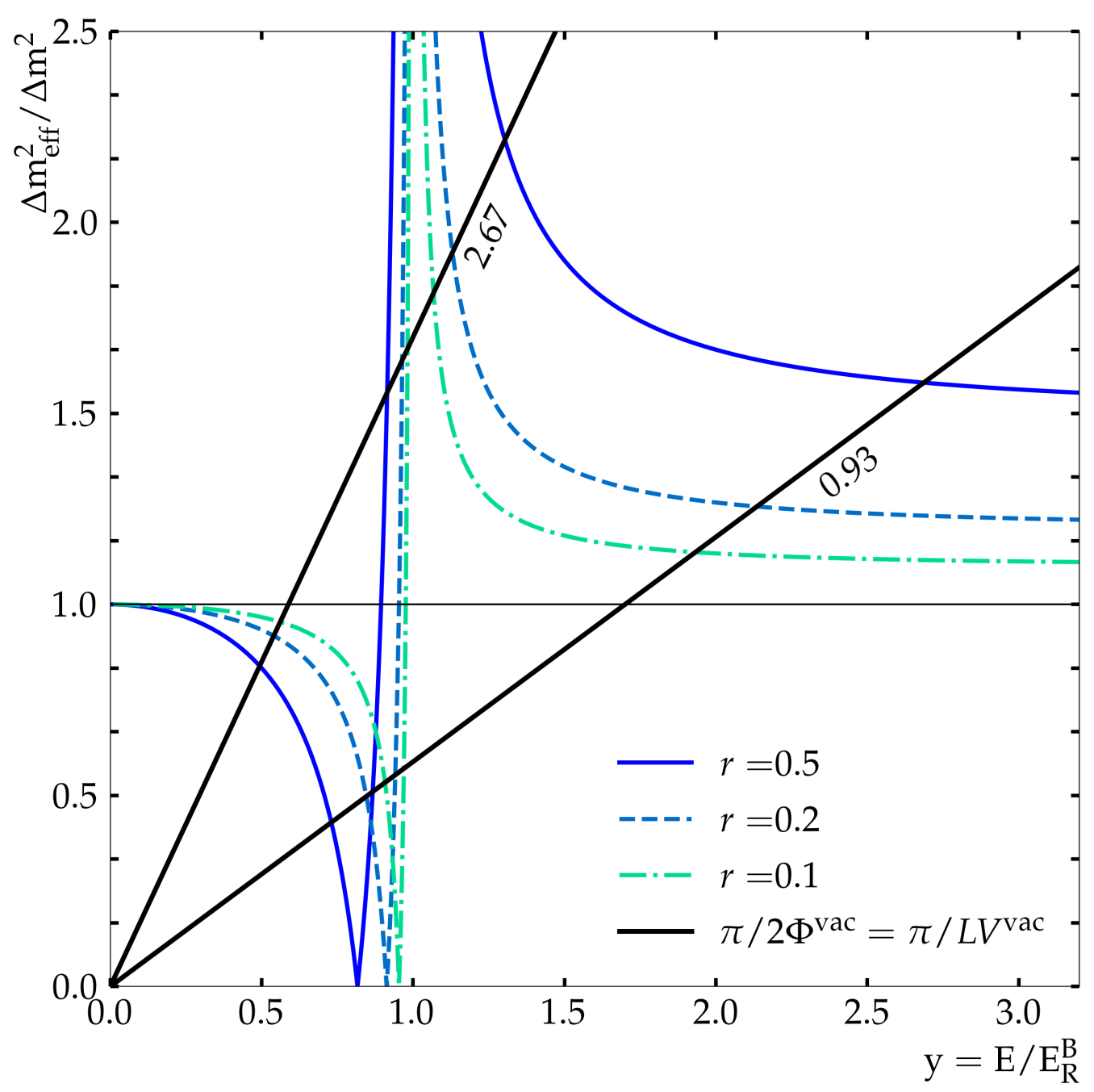

Figure 4. The effective mass splitting $\Delta m_{\text {eff }}^{2} / \Delta m^{2}$ as function of $y$ for different values of $r$. We take $\epsilon=0$. Shown are also lines $\pi / 2 L V^{\mathrm{vac}}(y)$ which correspond to two different values of baseline $\pi / 2 L V_{R}^{\mathrm{vac}}$ (numbers at the lines). Crossings of these lines with $\Delta m_{\text {eff }}^{2} / \Delta m^{2}$ give the points where the total phase $\Phi=\pi / 2$ (see text for explanations).

\subsection{Negative $\kappa$}

In the previous consideration we assumed that $\Delta m^{2}$ is positive, or more precisely, $\Delta m^{2}$ and $V^{B}=V_{2}^{B}-V_{1}^{B}$ are positive simultaneously. That is, the potentials follow the hierarchy of masses, which is automatically satisfied if both differences are given by $g_{2}^{2}-g_{1}^{2}$. As a consequence, $\kappa \geq 0$ and $r \geq 0$.

If, however, neutrinos have other sources of masses apart from VEV of $\phi$, the signs and values of $\Delta m^{2}$ and $V^{B}$ are independent. In this connection let us consider the case of negative $\kappa$ and $r$. Above the resonance the quantities $V^{B}$ and $V^{\text {vac }}$ have opposite signs. Therefore

1. The cancellation point $y_{c}$ (the MSW resonance on background) is above the refraction resonance: $y_{c}>1$. 
2. The ratio $R_{\Delta}$ increases from 1 at $y \rightarrow 0$ to maximum at $y \approx 1-\xi$ (no smearing).

3. The dip is above the resonance peak.

4. In asymptotics, $y \rightarrow \infty$, we have $R_{\Delta} \rightarrow 1-|r|<1$. So, one expects smaller value of $\Delta m_{\text {eff }}^{2}$ in comparison to the vacuum value: $\Delta m_{\text {eff }}^{2}=(1-|r|) \Delta m^{2}$.

\subsection{Phases and probabilities}

In the case of diagonal matrix of potentials in the neutrino mass basis the background potential modifies neutrino oscillations via an extra contributions to the oscillation phase:

$$
\Phi=\Phi^{\mathrm{vac}}+\Phi^{B}=\left(V^{\mathrm{vac}}+V^{B}\right) L,
$$

while the mixing is unchanged. Thus, for two neutrino mixing the $\nu_{\alpha}-\nu_{\beta}$ transition probability equals

$$
P_{\nu_{\alpha} \rightarrow \nu_{\beta}}(L, E)=\sin ^{2} 2 \theta \sin ^{2} 0.5 \Phi .
$$

We assume here constant density of background particles.

Since the phase $\Phi$ enters in the observables (probability) as $\cos \Phi$ or $\sin ^{2} \Phi / 2$, the change of sign of $V$ in the resonance does not lead to suppression due to integration over energy. (Notice that this is valid for $2 \nu$ case and without matter effect. In the $3 \nu-$ case we have interference of different channels with different frequencies and those terms are not even with respect to $V$.)

Observational effects of the background depend on the baseline of experiment. In figure 4 we show the lines

$$
\frac{\pi}{2 V^{\mathrm{vac}} L}=\frac{\pi}{2 \Phi^{\mathrm{vac}}}=\frac{\pi y}{2 V_{R}^{\mathrm{vac}} L} .
$$

For fixed $L$ the lines correspond to the inverse of the vacuum oscillation phase as function of $y$. With increase of $L$ the slope decreases. In figure 4 the left (right) line corresponds to the short (long) baseline.

The total oscillation phase equals

$$
\Phi=R_{\Delta}(y) \Phi^{\mathrm{vac}} .
$$

Therefore at crossings of $\pi /\left(2 \Phi^{\mathrm{vac}}(y)\right)$ and $R_{\Delta}(y)$ :

$$
\left|R_{\Delta}(y)\right|=\frac{\pi}{2 V^{\mathrm{vac} L}}
$$

we have

$$
\Phi\left(y^{\text {cross }}\right)=\pi / 2 \quad \rightarrow \quad \sin ^{2} 0.5 \Phi\left(y^{\text {cross }}\right)=0.5 .
$$

There are four crossings: low energy $y_{l}$, and $y_{-}, y_{+}$with left and right branches of the resonance peak as well at the resonance $y \approx 1$. The equation for crossing (3.17) can be written as

$$
y^{2}-1+r y(y-\epsilon)= \pm \frac{\pi}{2 V_{R}^{\operatorname{vac}} L} y\left(y^{2}-1\right) .
$$

For parts of the lines $R_{\Delta}(y)$, which are above the crossings the phase is big $\Phi>\pi / 2$, while for the parts below the crossings the phase is small: $\Phi<\pi / 2$. 
In figure 5 we show the oscillatory factor $\sin ^{2} 0.5 \Phi$ as function of $y$ for three different values of baseline. We performed smearing over energy.

The crossings determine four intervals of $y$ with different observational features.

- $y<y_{l}$ : the oscillatory curve with increasing period when $y \rightarrow 0$. At $y \rightarrow 0$ oscillations in background nearly coincide with the vacuum oscillations.

- $y_{l}<y<y_{-}$: oscillation dip. Here $\Phi<\pi / 2$, the background suppresses the phase.

- $y_{-}<y<y_{+}$: resonance interval. The phase is large: $\Phi>\pi / 2$. In the central resonance region $\Phi \gg 1$.

- $y>y_{+}$: tail at high energies, $\Phi<\pi / 2$, the phase decreases with increase of $y$.

With decreases of $L: y_{l} \rightarrow 0$, while $y_{-}, y_{+} \rightarrow 1$. Thus, the dip widens, whereas the resonance region becomes narrower.

\subsection{Bump: number of events}

The characteristic relevant for observations is not the width of the peak, but the energy range where the background effect is bigger than the standard oscillation effect. It is determined by the tails of resonance where $\left|V^{B}\right| \ll\left|V_{\max }^{B}\right|$. According to (2.32)

$$
\Phi^{B}=\Phi_{0} \frac{y-\epsilon}{y^{2}-1},
$$

with

$$
\Phi_{0} \equiv V_{0} L .
$$

As a criteria for strong effect, we can use $\sin ^{2} \Phi^{B} / 2=1 / 2$, which gives according to $(3.19)$

$$
y \approx 1 \pm \frac{\Phi_{0}}{\pi}(1-\epsilon) .
$$

Therefore the region of strong effect has width

$$
\Delta y=\frac{2 \Phi_{0}}{\pi}(1-\epsilon)
$$

This region decreases with increase of $\epsilon$.

For small $\Phi_{0}$ the background effect is small everywhere except for the resonance region. For instance, if $\Phi_{0}=\pi / 20$, then $\Delta y=0.2(\epsilon=-1)$. In the resonance region, $E=$ $E_{R}(1 \pm 0.1)$, we have $\sin ^{2} \Phi \geq 0.5$, while outside the resonance $\sin ^{2} \Phi \approx \sin ^{2} \Phi_{0}=0.024$

Let us consider total contribution from the resonance interval. Here the number of events is proportional to the integral

$$
I=\int_{y_{\min }}^{y_{\max }} d y \sin ^{2} 0.5 \Phi^{B}(y, \epsilon)=\int_{y_{\min }}^{y_{\max }} d y \sin ^{2} 0.5 \Phi_{0} \frac{y-\epsilon}{y^{2}-1},
$$

where $y_{\max }$ and $y_{\min }$ are determined by conditions the phase $\Phi^{B}\left(y_{\max }\right)=\Phi^{B}\left(y_{\min }\right)=\pi / 2$, so that $\sin ^{2} 0.5 \Phi^{B}=1 / 2$. We can approximate the oscillatory factor by its average value: $\sin ^{2} \Phi / 2 \approx 0.5$. Then

$$
I=0.5 \Delta y=\frac{\Phi_{0}}{\pi}(1-\epsilon)=(1-\epsilon) \frac{V_{0} L}{\pi},
$$

according to (3.19), and this result is valid for $\Phi_{0} / \pi \ll 1$. 

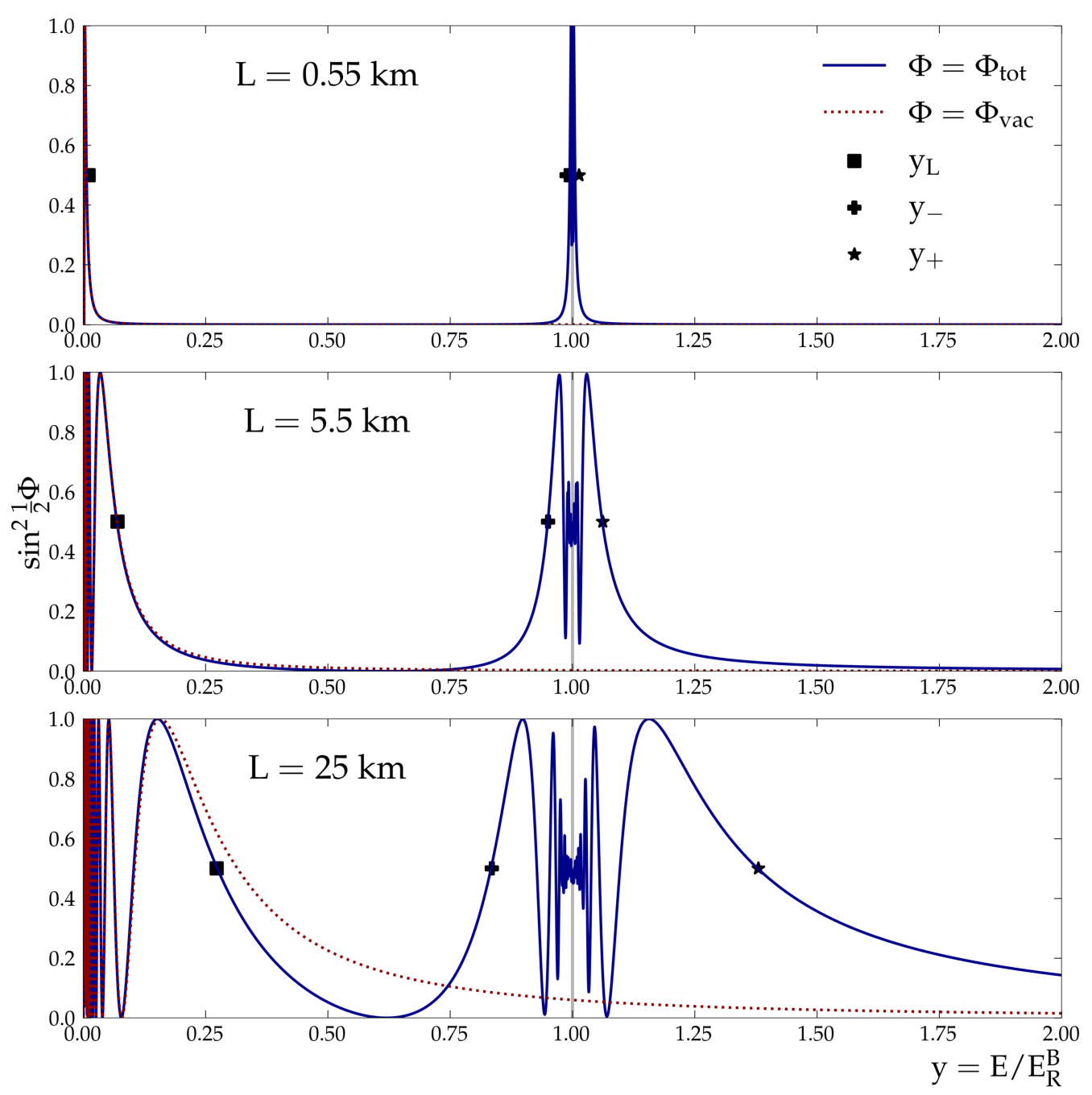

Figure 5. The oscillatory factor as function of energy $y$ for three different values of baselines $L$. We take $r=1.6$ and $\epsilon=0$. The dotted lines correspond to the oscillatory factors for pure vacuum oscillations $(r=0)$.

More precise computation of the integral (3.23) for any interval of $y$ can be done in the following way. Let us introduce $\delta_{y}$ (which depends on $\Phi_{0}$ ) such that in the range $|y-1|<\delta_{y}$ the phase is very big: $\Phi_{0} / 2 \delta_{y} \gg 1$, and consequently, the sine has very fast oscillations $\left(\delta_{y} \ll \Delta y\right)$. Then the integral $I$ can be split in three parts: with integration over $y$ in the intervals $\left[1-\delta_{y} \div 1+\delta_{y}\right],\left[0 \div 1-\delta_{y}\right]$ and $\left[1+\delta_{y} \div \infty\right]$. In the first (central) interval the integrand can be approximated by $1 / 2$, and consequently,

$$
I=\delta_{y}+\int_{0}^{1-\delta_{y}} d y \sin ^{2} \frac{0.5 \Phi_{0}(y-\epsilon)}{y^{2}-1}+\int_{1+\delta_{y}}^{\infty} d y \sin ^{2} \frac{0.5 \Phi_{0}(y-\epsilon)}{y^{2}-1} .
$$

The tail integrals can be computed numerically as follows.

In the central (resonance) region, $-2 \Phi_{0} / \pi<(y-1)<2 \Phi_{0} / \pi$, we can substitute the integrand $\sin ^{2} \Phi / 2$ by $1 / 2$. Outside the resonance region, $0<y<1-2 \Phi_{0} / \pi$ (lower region) 
and $y>1+2 \Phi_{0} / \pi$ (upper region), the sine squared can be approximated by

$$
\frac{1}{2}\left(\frac{2 \Phi_{0}}{\pi}\right)^{2} \frac{(y-\epsilon)^{2}}{\left(y^{2}-1\right)^{2}}
$$

normalized in such a way that at the borders it equals $1 / 2$. Then for small $\Phi_{0} / \pi$ the high and the low energy tails give

$$
I_{h} \approx \frac{\Phi_{0}}{\pi} \frac{1-\epsilon}{2}, \quad I_{l} \approx \frac{\Phi_{0}}{\pi} \frac{1-\epsilon}{2}\left(1-\frac{2 \Phi_{0}}{\pi}\right)
$$

and the sum equals

$$
I_{\text {tail }}=I_{h}+I_{l} \approx \frac{2 \Phi_{0}}{\pi} \frac{1-\epsilon}{2} .
$$

The ratio of the tails to the resonance (3.24) contributions equals

$$
\frac{I_{\text {tail }}}{I_{c}} \approx 1-\mathcal{O}\left(\frac{\Phi_{0}}{\pi}\right)
$$

and it depends on the phase weakly: with increase of $\Phi_{0}$ the ratio decreases. The contribution from resonance width (2.22) is negligible.

\subsection{Adding usual matter effect}

The matter potential $V_{e}=\sqrt{2} G_{F} n_{e}$ does not depend on energy in the range we are considering. The equality $V_{e} \approx V^{\mathrm{vac}}$ determines the MSW resonance energy $E^{\mathrm{MSW}}$. Since in this setup the mixing does not change by the background, the MSW resonance condition has usual form:

$$
\frac{\Delta m_{\mathrm{eff}}^{2}(E)}{2 E}=\frac{V_{e}}{\cos 2 \theta} .
$$

There are three possibilities depending on relative values of $V_{e}$ and $V_{R}^{\mathrm{vac}}$.

I. $V_{e}<V_{R}^{\mathrm{vac}}$ : in this case the refraction resonance is below the MSW resonance $E_{R}^{B}<$ $E^{\mathrm{MSW}}$ (see figure 3). There are three crossing of $V^{\mathrm{vac}}(y)$ with $V_{e}$ in the neutrino channel:

(i) Standard MSW resonance. It is shifted to higher energies due to background contribution. The resonance energy with background correction can be found from eq. (3.28). The expression is simplified in the case $y^{\mathrm{MSW}} \gg 1$, so that we can take the asymptotic value $\Delta m_{\text {eff }}^{2}(E) \approx \Delta m^{2}(1+r)$. As a result,

$$
E^{\mathrm{MSW}}=E^{\mathrm{MSW}, 0}(1+r),
$$

where $E^{\mathrm{MSW}, 0}$ is the standard resonance energy without background:

$$
E^{\mathrm{MSW}, 0}=\cos 2 \theta \frac{\Delta m^{2}}{2 V_{e}} .
$$

The shift of MSW resonance can be used to search for the background effect.

(ii) New crossing near refraction resonance, $y \approx 1$.

(iii) New crossing with the low energy branch of $V^{\text {tot }}$. 
In the $\bar{\nu}$ - channel there are two crossings: (i) near the refraction resonance; (ii) with low energy branch of $V^{\text {sum }}$.

In the crossing points the mixing in medium (matter plus background) becomes maximal.

If $V_{e} \ll V_{R}^{\mathrm{vac}}, E_{R}^{B} \ll E^{\mathrm{MSW}}$, at low energies and short baseline experiments the effects of four new crossings become unobservable because in these crossings $\Phi \ll 1$.

II. $V_{e}>V_{R}^{\text {vac: }}$ in this case the refraction resonance is above the MSW resonance: $E_{R}^{B}>E^{\mathrm{MSW}}$. Depending on $\epsilon$ the shift of the MSW resonance can be to higher or low energies.

As before there are two new crossings in the $\nu$-channel and two new crossings in the $\bar{\nu}$-channel. In the $\nu$-channel one crossing is near the refraction resonance, and another one is in the high energy branch. The energy of the latter can be substantially larger than $y=1$. In the $\bar{\nu}$-channel the two crossings are near the refraction resonance being in the low energy branch.

III. The case of $V_{e} \approx V_{R}^{\mathrm{vac}}$ is of special interest: the standard MSW resonance coincides with the refraction resonance, while two new resonances (at $y>1$ and at $y<1$ ) can be far from the refraction resonance $y=1$.

\subsection{Generation of mixing in the background}

In the previous consideration the matrix of potentials had only one entry and so it was diagonal in the mass eigenstate basis. If couplings of other mass states with background are not neglected the transition $\nu_{1} \bar{\chi} \rightarrow \nu_{2} \bar{\chi}$ generates a non-diagonal element of the matrix of potentials which is proportional $g_{1} g_{2}^{*}$. In the $2 \nu$ case the total Hamiltonian becomes

$$
H^{B}=\left(\begin{array}{cc}
0 & \alpha V^{B} \\
\alpha^{*} V^{B} & V^{\mathrm{vac}}+V^{B}
\end{array}\right)=V^{\mathrm{vac}}\left(\begin{array}{cc}
0 & \alpha \kappa \\
\alpha^{*} \kappa & 1+\kappa
\end{array}\right),
$$

where

$$
\alpha \equiv \frac{g_{1} g_{2}^{*}}{\left|g_{2}\right|^{2}-\left|g_{1}\right|^{2}}
$$

$V^{B}=V^{B}\left(|g|^{2} \rightarrow\left|g_{2}\right|^{2}-\left|g_{1}\right|^{2}\right)$ and $V^{B}\left(|g|^{2}\right)$ is the background potential discussed in the previous sections. $\kappa$ is defined in (3.2).

Notice that the resonance energies are different for different neutrino mass states $\nu_{i}$ :

$$
E_{2 R}-E_{1 R}=\frac{\Delta m^{2}}{2 m_{\chi}} \ll E_{R}
$$

but this difference is still much smaller than the scale of smearing due to motion of scatterers. Therefore we can neglect dependence of potentials on the neutrino masses and the only relevant dependence on type of neutrino is in the coupling constants.

Diagonalization of the matrix (3.30) gives the difference of eigenvalues

$$
R_{\Delta}=\sqrt{(1+\kappa)^{2}+(2 \alpha \kappa)^{2}}
$$


and the mixing angle of mass states

$$
\sin ^{2} 2 \theta^{B}=\frac{(2 \alpha \kappa)^{2}}{(1+\kappa)^{2}+(2 \alpha \kappa)^{2}} .
$$

The flavor mixing angle becomes

$$
\theta_{f}=\theta+\theta^{B}
$$

Let us consider different limits and benchmark points.

1. $y \rightarrow 0: R_{\Delta} \rightarrow 1$ and $\theta^{B} \rightarrow 0$. The background effect is negligible.

2. $y \rightarrow y_{c}$ : the cancellation point $\left(V^{B}=-V^{\mathrm{vac}}\right)$ becomes the energy of MSW resonance on the background. Here the mixing is maximal $\sin ^{2} 2 \theta^{B}=1$ and splitting is non-zero:

$$
R_{\Delta}=2 \alpha .
$$

The transition probability equals $P \approx \sin ^{2}\left(\alpha \Phi^{\mathrm{vac}}\right)$.

3. In the peak, $y \approx 1: V^{B} \gg V^{\text {vac }}$, the ratio equals

$$
R_{\Delta}=\frac{V^{B}}{V^{\mathrm{vac}}} \sqrt{1+4 \alpha^{2}}=\kappa \sqrt{1+4 \alpha^{2}},
$$

and the angle is

$$
\sin ^{2} 2 \theta^{B}=\frac{4 \alpha^{2}}{1+4 \alpha^{2}}
$$

4. In the refraction resonance, $y=1\left(V^{B}=0\right): R_{\Delta}=1$ and $\theta^{B}=0$.

5. In asymptotics $y \rightarrow \infty: V^{B} / V^{\text {vac }} \rightarrow r$. Correspondingly,

$$
R_{\Delta}=\sqrt{(1+r)^{2}+(2 \alpha r)^{2}},
$$

and

$$
\sin ^{2} 2 \theta^{B}=\frac{(2 \alpha r)^{2}}{(1+r)^{2}+(2 \alpha r)^{2}}
$$

The transition probability equals

$$
P=\sin ^{2} 2\left(\theta+\theta^{B}\right) \sin ^{2}\left(\Phi^{\mathrm{vac}} R / 2\right) .
$$

For small $\alpha$ in comparison to no-mixing case modifications of $P$ are small. The most significant change is in the cancellation region.

Finally, let us comment on the case of three different fermions $\chi_{j}$ - each per generation. If $\mathrm{VEV}$ of $\phi$ is the only source of neutrino mass then the couplings are diagonal in the mass basis. Furthermore, transition $\nu_{i} \bar{\chi}_{i} \rightarrow \nu_{j} \bar{\chi}_{j}$ will not form potential, since final $\bar{\chi}_{j}$ differs from initial $\bar{\chi}_{i}$ being orthogonal each other. In this case the matrix of potentials is diagonal and the difference of diagonal elements, $V=V_{i}-V_{j} \propto\left|g_{i}^{2}\right|-\left|g_{j}^{2}\right|$, will enter expressions considered above. 


\subsection{Resonance refraction and Glashow resonance}

In the standard model, the resonance refraction is realized in the Glashow resonance: that is, in the $\nu_{e}-e$ scattering with $W$ boson as the mediator. The resonance energy equals $E_{R}=m_{W}^{2} / 2 m_{e} \approx 6.4 \mathrm{PeV}$. Dependence of the potential on neutrino energy is described by the eq. (2.14) with $\epsilon=-1, V_{0}=\sqrt{2} G_{F} n_{e}$ and $\xi=3 g_{W}^{2} / 16 \pi$.

At low energies the potential coincides with the Wolfenstein potential. The difference from what we have discussed before is that the coupling is large $g_{W} \sim \mathcal{O}(1)$. Therefore the width of the resonance is not negligible, enhancement is not extremely strong and smearing effect is weaker. The maxima

$$
\left|V_{\max }\right|=V_{0} \frac{m_{W}}{\Gamma_{W}}=V_{0} \frac{16 \pi}{3 g_{W}^{2}}
$$

are achieved at $E=E_{R}\left(1 \pm \Gamma_{W} / m_{W}\right)$. In the resonance region the vacuum contribution, $\Delta m^{2} / 2 E$ is negligible: $r=10^{-6}$. Vacuum mixing is strongly suppressed. Furthermore, dumping due to absorption can be substantial.

The refraction length in resonance can be reduced by factor 20 in comparison to the Wolfenstein length, being of the order $300 \mathrm{~km}$. However, existence of observable effects at the Earth is questionable.

1. Oscillation effects with usual $\Delta m^{2}$ and $\theta$ are negligible. Refraction index is still very close to 1 , so that bending and refraction effects are negligible too.

2. One can explore possible effect in astrophysical objects - sources of high energy neutrinos.

3. Mixing of active neutrinos with sterile neutrinos of mass $10^{2} \mathrm{eV}$ can be considered. In this case $\Delta m^{2} / 2 E_{R} \sim V_{e}$ and the mixing can be enhanced in matter.

\section{Applications to specific experiments}

\subsection{Signatures and implications}

Recall that the oscillatory pattern in terms of universal variables, $R_{\Delta}(y)$ and $y$ depends on (i) $r$ - relative strength of interactions with background (3.3), (ii) $\epsilon$ - charge asymmetry of the background, (iii) baseline $L$. Thus, observing the oscillatory pattern at given $L$ one can determine $\epsilon$ and $r$ (which is the combination of the fundamental parameters and density of a background (3.3)) or put bounds on these parameters.

Observable effects of the background vanish completely if $r \rightarrow 0$, however, they do not disappear when $\epsilon \rightarrow 1$. At $\epsilon=1$ the resonance is absent, the cancellation point is at $y_{c}=1$ and

$$
R_{\Delta}(y)=1+r \frac{y}{y+1} .
$$

So, the corrections increase with $y$ : at $y=1$ the ratio equals $R_{\Delta}=1+r / 2$, for $y \rightarrow \infty$ : $R_{\Delta}=1+r$. For large energies the background effects are determined by $r$ and dependence on $\epsilon$ is weak. 
To some extend the effects of $\epsilon$ and $r$ on the oscillatory pattern correlate, and there is certain degeneracy. However, variations of the pattern with $r$ can be much more substantial than that with $\epsilon$. Effect of $\epsilon$ is restricted by its minimal value -1 .

The presence of the resonance bump testifies for $\epsilon \neq 1$. Value of $\epsilon$ determines the benchmark energies. With $\epsilon \rightarrow-1$ the region of distortions in the resonance interval becomes wider. Measuring the oscillatory pattern in different energy ranges allows to disentangle effects of $r$ and $\epsilon$. Let us summarize signatures of interactions with background. For $\kappa>0$ they include:

- deviation of the oscillatory pattern from $\sin ^{2}(A / y)$ in the low energy interval;

- oscillation dip at $y<1$, with zero at $y_{c}$;

- increase of the probability at $y \rightarrow 1$;

- bump at $y \sim 1$;

- tail at $y>1.2$, which corresponds to larger $\Delta m_{\text {eff }}^{2}$ than at low energies.

In the presence of usual matter we have in addition

- shift of the MSW resonance to larger (if $E^{\mathrm{MSW}}>E_{R}^{B}$ ) or smaller (if $E^{\mathrm{MSW}}<E_{R}^{B}$ ) energies;

- appearance of new MSW resonances.

For $\kappa<0$ the dip is at higher energies. In asymptotics the effective $\Delta m_{\text {eff }}^{2}$ is smaller than that at low energies.

Thus, to search for effects for fixed $L$ one can consider different energy intervals. For a given neutrino beam one can use different $L$, e.g., results from near and far detectors.

\subsection{MiniBooNE excess and resonance refraction}

The low energy excess of events reported by the MiniBooNE collaboration [7] could be a manifestation of the resonance refraction [6]. The background is composed of the overdense relic neutrinos. In this case $m_{\chi}=0.05 \mathrm{eV}$ and $\epsilon \approx 0$.

The best fit of the MiniBooNE data is obtained for values of parameters

$$
E_{R}^{B}=(320-340) \mathrm{MeV}, \quad Y \equiv \frac{g^{2}\left(n_{\chi}+\bar{n}_{\chi}\right)}{8 m_{\chi}} \geq 10^{-3} \mathrm{eV}^{2} .
$$

Then

$$
m_{\phi}=\sqrt{2 m_{\chi} E_{R}^{B}}=5.8 \mathrm{keV} .
$$

Notice that the mediator and background particles are light enough and therefore the astrophysical bounds on $g$ are applicable (see section 2.1).

From (4.2) we obtain

$$
V_{0}^{B}=\frac{2 Y}{E_{R}^{B}}=5.9 \cdot 10^{-12} \mathrm{eV}, \quad V_{R}^{\mathrm{vac}}=3.7 \cdot 10^{-12} \mathrm{eV},
$$


and correspondingly,

$$
r=\frac{4 Y}{\Delta m^{2}}=1.59, \quad y_{c}=0.62 .
$$

Thus, in the resonance region and above it the background potential dominates. The usual matter potential is very small: $V_{e}=2 \cdot 10^{-13} \mathrm{eV}$.

The MiniBooNE baseline $L_{\mathrm{MB}}=541 \mathrm{~m}$ corresponds to

$$
\frac{1}{L_{\mathrm{MB}}}=3.1 \cdot 10^{-10} \mathrm{eV} \gg V_{R}^{\mathrm{vac}}, V_{0}^{B},
$$

which means that the phase is very small, $\Phi \ll 1$, everywhere except for narrow region close to $y=1$. The resonance peak is smeared by the energy resolution.

Let us show that this solution is excluded because of strong dependence of the effective $\Delta m_{\text {eff }}^{2}$ on energy $(y)$. In figure 6 we show $\Delta m_{\text {eff }}^{2}$ as function of energy for $E_{R}=320 \mathrm{MeV}$, $\epsilon=0$ and different values of $r$. At low energies $y \ll 1, \Delta m_{\text {eff }}^{2} \approx \Delta m^{2}$ (as in vacuum) while above the resonance

$$
\Delta m_{\mathrm{eff}}^{2} \approx\left(1+r \frac{y^{2}}{y^{2}-1}\right) \Delta m^{2}
$$

According to this equation for $y=2$ and $y=3$, which correspond to $E=680 \mathrm{MeV}$ and $1020 \mathrm{MeV}$, the enhancement of $\Delta m_{\text {eff }}^{2}$ is given by factors 3.12 and 2.79 respectively. In asymptotics, $y \rightarrow \infty$, it converges to 2.59 .

Figure 6 shows also results of measurements of the "atmospheric" $\Delta m^{2} \approx m_{3}^{2}$ at different energies. At the lowest energies, $E=(2-5) \mathrm{MeV}\left(y \sim 10^{-2}\right)$ the data on $\Delta m_{e e}^{2} \approx \Delta m_{31}^{2}$ are provided by the reactor experiments [33-35]. Here the background effect can be neglected. The T2K experiment $[36,37]$ measures $\Delta m_{32}^{2}$ at $(0.3-1.3) \mathrm{GeV}$ which is slightly above the resonance. At higher energies (essentially in asymptotics) the data are given by NOvA [38] and then MINOS and MINOS+ [39]. At even higher energies IceCube DeepCore [40] and ANTARES [41] give information on $\Delta m_{32}^{2}$.

The main conclusion is that within the experimental error bars $\Delta m_{\text {eff }}^{2}$ does not depend on energy over 4 orders of magnitude. This puts strong bound on strength of interaction with background:

$$
r \lesssim 0.01
$$

which certainly excludes $r>1.6$ required by MiniBooNE explanation.

Similar result can be obtained for negative $r$. Now above the resonance the predicted values of $\Delta m_{\text {eff }}^{2}$ are below the experimental points.

The same consideration with the same conclusion is applied for the bosonic background and fermionic mediator. In particular, the figure 6 will be unchanged. The only difference is that the potential is 2 times larger which can be accounted by renormalization $g \rightarrow \sqrt{2} g$. The latter could have some implications to particle physics model but not to the exclusion.

\subsection{Bounds on the background effects}

We have obtained the upper bound on strength of the background effects $r$ (4.6) for $E_{R} \sim$ $320 \mathrm{MeV}$. According to figure 6 similar bound can be established in the interval of $E_{R}$ from $10 \mathrm{MeV}$ to $10 \mathrm{GeV}$. For $E_{R}<1 \mathrm{MeV}$ - no distortion is expected in the region of 


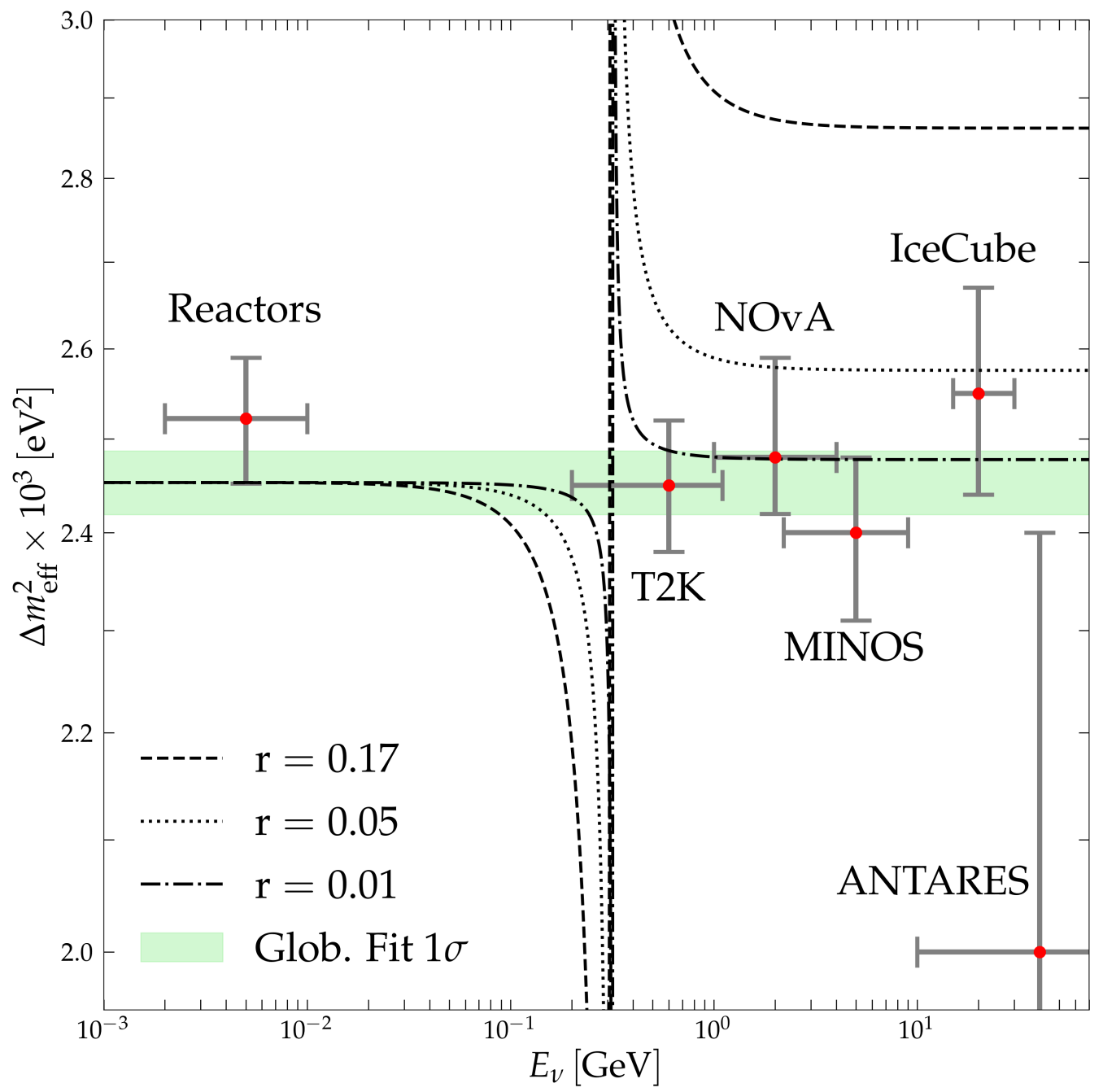

Figure 6. The effective mass squared difference as function of the neutrino energy for different values or $r$. The curves are normalized at $E \rightarrow 0$ to the value of the $\Delta m_{32}^{2}$ from the global fit of all the data. Explanation of the MiniBooNE requires $r>1$. Shown are the values of $\Delta m_{32}^{2}, \Delta m_{31}^{2}$ and $\Delta m_{e e}^{2}$ extracted from experiments at different energies.

observations (i.e. at $E>\mathrm{MeV}$ ), while for $E_{R}>10 \mathrm{GeV}$ the effect of background in the observable region becomes much smaller than vacuum effect and it decreases with energy decrease.

The strength $r$ (3.3) can be written as

$$
r=\frac{2 V_{0} E_{R}}{\Delta m^{2}}
$$

This means that for given $E_{R}$ and $r$ the potential is restricted by

$$
V_{0}=r \frac{\Delta m^{2}}{2 E_{R}} .
$$

The largest value of $E_{R}$, for which a given bound on $r$ exists, gives the most strong restric- 
tion on $V_{0}$. Therefore according to (4.7)

$$
r(E=0.32 \mathrm{GeV})=r(E=3 \mathrm{GeV}) \frac{0.32 \mathrm{GeV}}{3 \mathrm{GeV}} \approx 10^{-3} .
$$

Thus, consideration at higher energies allows to strengthen the bound on $r$.

For the background particles at rest the strength factor can be written as

$$
r=\frac{g^{2} n_{\chi}}{2 m_{\chi} \Delta m^{2}}
$$

Is the bound on $r$ we obtained from resonance refraction substantial, or there are other more strong bounds? One such a bound on the system comes from contribution of $\chi$ to the dark matter in the Universe:

$$
\rho_{\chi}=E_{\chi} n_{\chi} \geq m_{\chi} n_{\chi}
$$

For a given value of $m_{\chi}$ this gives the number density of $\chi$ which compose $\rho_{\chi} / \rho_{\text {DM }}$ fraction of the local dark matter:

$$
n_{\chi} \sim \frac{\rho_{\mathrm{DM}}}{m_{\chi}} \frac{\rho_{\chi}}{\rho_{\mathrm{DM}}} .
$$

Inserting this expression into (4.10) and taking for the local energy density of DM $\rho_{\mathrm{DM}}=$ $0.4 \mathrm{GeV} / \mathrm{cm}^{3}$, we obtain the strength factor

$$
r=2.6 \cdot 10^{-7}\left(\frac{g^{2}}{10^{-3}}\right)^{2}\left(\frac{0.05 \mathrm{eV}}{m_{\chi}}\right)^{2}\left(\frac{\rho_{\chi}}{\rho_{\mathrm{DM}}}\right) .
$$

For $g$ satisfying the bound (2.2), $\rho_{\chi}=\rho_{\mathrm{DM}}$ and $m_{\chi}=0.05 \mathrm{eV}$ eq. (4.13) gives $r=$ $2.6 \cdot 10^{-14}$ which is much below the refraction bound. For these values of parameters $n_{\chi}=8 \cdot 10^{9} \mathrm{~cm}^{-3} \cdot r$ can be enhanced if we take smaller mass of $\chi$ and $g=10^{-3}$, which satisfies the laboratory bounds but requires more complicated cosmological evolution that allows to avoid BBN and CMB bounds. Then $r=10^{-3}$ can be obtained for $m_{\chi}=8 \cdot 10^{-4} \mathrm{eV}$. The corresponding number density of $\chi$ equals $n_{\chi}=5 \cdot 10^{11} \mathrm{~cm}^{-3}$.

This consideration is valid for bosonic background with changing subscripts $\chi \leftrightarrow \phi$ in eq. (4.12)-(4.13). For the fermionic background additional restrictions follow from Pauli principle. Indeed, the density indicated above gives the Fermi momentum of the degenerate gas $p_{F}=\left(6 \pi^{2} n_{\chi}\right)^{1 / 3}=1.3 \mathrm{eV}$. That is, $E_{\chi} \approx p_{F} \gg m_{\chi}$, and therefore we deal here with strongly degenerate fermion gas. Consequently, in all considerations above we should substitute

$$
m_{\chi} \rightarrow E_{\chi} \sim p_{F}=\left(6 \pi^{2} n_{\chi}\right)^{1 / 3} .
$$

In particular, $E_{R}=m_{\phi}^{2} / 2 E_{\chi}$ and

$$
r=\frac{g^{2}}{2 \Delta m^{2}} \frac{n_{\chi}}{E_{\chi}}=\frac{g^{2}}{\left.2\left(6 \pi^{2}\right)^{1 / 3}\right) \Delta m^{2}}\left(n_{\chi}\right)^{2 / 3} .
$$

Using expression for the energy density in $\chi$

$$
\rho_{\chi}=E_{\chi} n_{\chi}=\left(6 \pi^{2}\right)^{1 / 3} n_{\chi}^{4 / 3}
$$


we obtain

$$
r=\frac{g^{2} \sqrt{\rho_{\chi}}}{2 \sqrt{6} \pi \Delta m^{2}}
$$

Numerically this gives

$$
r=4.7 \cdot 10^{-8}\left(\frac{g^{2}}{10^{-3}}\right)^{2} \sqrt{\frac{\rho_{\chi}}{\rho_{\mathrm{DM}}}} .
$$

Thus, $r$ is determined by the coupling constant and fraction of the DM in $\chi$ and does not depend on $m_{\chi}$. The value $r \leq 4.7 \cdot 10^{-8}$, which is much smaller than sensitivity range to the resonance refraction effects of experiments at the laboratory energies.

The bound 4.17 can be relaxed if $\chi$ are not distributed uniformly in the Galaxy but in clouds (the $\chi$-stars) of size $d$ with spacing $l$. In this case, the density in the clouds increases as $(l / 2 r)^{3}$, and taking into account the dependence of $E_{\chi}$ on $n_{\chi}$, we find that the energy density $\rho_{\chi}$ increases as $(l / 2 r)^{4}$. Correspondingly, the ratio in 4.17 increases as $(l / 2 r)^{2}$. The value $r \sim 10^{-3}$ would require the structure with voids between the $\chi$-stars 200 times bigger than the size of the stars. Clearly, this clustering does not remove the bound obtained from oscillation experiments in section 4.2 .

\section{Conclusions}

1. In general, the medium potential is function of the neutrino energy and this function depends on the C-asymmetry of a background. The energy dependence of $V^{B}$ may have a resonance character related to the exchange of (on shell) mediator of interactions. Resonance is realized at $\sqrt{s}=M_{\text {med }}$ and for light mediators and light scatterers (which requires extension of the Standard model) the resonance refraction can occur at energies available at laboratories.

2. The relative correction to the vacuum (kinetic) term from background vanishes at low energies, it can dominate in resonance and above it. At high energies the correction converges to constant. The interplay of the energy dependent potential $V^{B}(y)$ and vacuum contribution $V^{\mathrm{vac}}(y)$ has several important features:

- Cancellation of the contributions which corresponds to the MSW resonance on background (when mixing in the background is introduced),

- above the resonance $V^{B}(y)$ gives correction to $V^{\text {vac }}(y)$ which does not disappear in asymptotics $E \rightarrow \infty$.

3. The background can produce mixing of mass states, that is, the non-diagonal matrix of potentials in the mass basis. For small mixing substantial effect on oscillations appears in the region around the cancellation point (the MSW resonance on a background).

4. For long-baseline experiments usual matter effect should be added. The interaction with background shifts the energy of MSW resonance (which provides important signature) and leads to appearance of new resonances around $E_{R}^{B}$. 
5. Signatures of refraction on the background include: (i) deviation of the oscillatory pattern in energy from $\sin ^{2}(A / E)$, (ii) dip of the oscillation probability below or above resonance, (iii) bump in the resonance region, (iv) additional contribution to $V^{\mathrm{vac}}(y)$ above refraction resonance which does not disappear in asymptotics.

6. Effects of background can be considered as modification of the effective $\Delta m_{\text {eff }}^{2}(y)$ with peculiar dependence on energy.

7. As an example we applied our results to the MiniBooNE excess interpreted as bump produced by the refraction resonance. We show that this interpretation is excluded because of strong difference of $\Delta m_{\text {eff }}^{2}$ expected at high energies (T2K, NOvA, MINOS, MINOS+, IceCube, ANTARES) and low energies (reactor experiments) in contrast to observations. We obtain the bound on the relative strength of neutrino interactions with background $r<(0.001-0.01)$.

\section{Acknowledgments}

A.Y.S. thanks E. Kh. Akhmedov for useful discussions. V.B.V. is grateful for support from the Villum Fonden through the project no. 29388, and the ICTP Postgraduate Diploma Programme.

Open Access. This article is distributed under the terms of the Creative Commons Attribution License (CC-BY 4.0), which permits any use, distribution and reproduction in any medium, provided the original author(s) and source are credited.

\section{References}

[1] L. Wolfenstein, Neutrino oscillations in matter, Phys. Rev. D 17 (1978) 2369 [INSPIRE].

[2] R. Opher, Coherent scattering of cosmic neutrinos, Astron. Astrophys. 37 (1974) 135 [INSPIRE].

[3] V.D. Barger, K. Whisnant, S. Pakvasa and R.J.N. Phillips, Matter effects on three-neutrino oscillations, Phys. Rev. D 22 (1980) 2718 [InSPIRE].

[4] P. Langacker, J.P. Leveille and J. Sheiman, On the Detection of Cosmological Neutrinos by Coherent Scattering, Phys. Rev. D 27 (1983) 1228 [INSPIRE].

[5] C. Lunardini and A. Smirnov, The minimum width condition for neutrino conversion in matter, Nucl. Phys. B 583 (2000) 260.

[6] J. Asaadi, E. Church, R. Guenette, B.J.P. Jones and A.M. Szelc, New light Higgs boson and short-baseline neutrino anomalies, Phys. Rev. D 97 (2018) 075021 [arXiv:1712.08019] [INSPIRE].

[7] MiniBooNE collaboration, Updated miniboone neutrino oscillation results with increased data and new background studies, Phys. Rev. D 103 (2021) 052002 [arXiv:2006.16883].

[8] J.F. Nieves and S. Sahu, Neutrino effective potential in a fermion and scalar background, Phys. Rev. D 98 (2018) 063003 [arXiv:1808.01629] [INSPIRE]. 
[9] J.F. Nieves and S. Sahu, Neutrino damping in a fermion and scalar background, Phys. Rev. D 99 (2019) 095013 [arXiv: 1812.05672] [INSPIRE].

[10] S.-F. Ge and S.J. Parke, Scalar nonstandard interactions in neutrino oscillation, Phys. Rev. Lett. 122 (2019) 211801 [arXiv:1812.08376] [INSPIRE].

[11] K.-Y. Choi, E.J. Chun and J. Kim, Neutrino oscillations in dark matter, Phys. Dark Univ. 30 (2020) 100606 [arXiv:1909.10478] [INSPIRE].

[12] K.S. Babu, G. Chauhan and P.S. Bhupal Dev, Neutrino nonstandard interactions via light scalars in the Earth, Sun, supernovae, and the early Universe, Phys. Rev. D 101 (2020) 095029 [arXiv: 1912.13488] [INSPIRE].

[13] K.-Y. Choi, E.J. Chun and J. Kim, Dispersion of neutrinos in a medium, arXiv:2012.09474 [INSPIRE].

[14] S.-F. Ge and H. Murayama, Apparent CPT violation in neutrino oscillation from dark non-standard interactions, arXiv: 1904.02518 [INSPIRE].

[15] A. Berlin, Neutrino oscillations as a probe of light scalar dark matter, Phys. Rev. Lett. 117 (2016) 231801 [arXiv: 1608.01307] [INSPIRE].

[16] W. Rodejohann, X.-J. Xu and C.E. Yaguna, Distinguishing between Dirac and Majorana neutrinos in the presence of general interactions, JHEP 05 (2017) 024 [arXiv:1702.05721] [INSPIRE].

[17] M. Lindner, F.S. Queiroz, W. Rodejohann and X.-J. Xu, Neutrino-electron scattering: general constraints on $Z^{\prime}$ and dark photon models, JHEP 05 (2018) 098 [arXiv: 1803.00060] [INSPIRE].

[18] G. Arcadi, M. Lindner, J. Martins and F.S. Queiroz, New physics probes: atomic parity violation, polarized electron scattering and neutrino-nucleus coherent scattering, Nucl. Phys. B 959 (2020) 115158 [arXiv: 1906.04755] [INSPIRE].

[19] M. Lindner, W. Rodejohann and X.-J. Xu, Coherent neutrino-nucleus scattering and new neutrino interactions, JHEP 03 (2017) 097 [arXiv: 1612.04150] [INSPIRE].

[20] Y. Farzan, M. Lindner, W. Rodejohann and X.-J. Xu, Probing neutrino coupling to a light scalar with coherent neutrino scattering, JHEP 05 (2018) 066 [arXiv:1802.05171] [INSPIRE].

[21] V. Brdar, W. Rodejohann and X.-J. Xu, Producing a new fermion in coherent elastic neutrino-nucleus scattering: from neutrino mass to dark matter, JHEP 12 (2018) 024 [arXiv: 1810.03626] [INSPIRE].

[22] J.D. Bjorken, R. Essig, P. Schuster and N. Toro, New fixed-target experiments to search for dark gauge forces, Phys. Rev. D 80 (2009) 075018 [arXiv:0906.0580] [InSPIRE].

[23] B. Batell, M. Pospelov and A. Ritz, Exploring portals to a Hidden sector through fixed targets, Phys. Rev. D 80 (2009) 095024 [arXiv:0906.5614] [INSPIRE].

[24] R. Essig, R. Harnik, J. Kaplan and N. Toro, Discovering new light states at neutrino experiments, Phys. Rev. D 82 (2010) 113008 [arXiv: 1008.0636] [INSPIRE].

[25] BaBAR collaboration, Search for a dark photon in $e^{+} e^{-}$collisions at BaBar, Phys. Rev. Lett. 113 (2014) 201801 [arXiv:1406.2980] [INSPIRE].

[26] BABAr collaboration, Search for a muonic dark force at BaBar, Phys. Rev. D 94 (2016) 011102 [arXiv: 1606.03501] [INSPIRE]. 
[27] R. Harnik, J. Kopp and P.A.N. Machado, Exploring nu signals in dark matter detectors, JCAP 07 (2012) 026 [arXiv: 1202.6073] [INSPIRE].

[28] E.G. Adelberger, B.R. Heckel, S.A. Hoedl, C.D. Hoyle, D.J. Kapner and A. Upadhye, Particle physics implications of a recent test of the gravitational inverse sqaure law, Phys. Rev. Lett. 98 (2007) 131104 [hep-ph/0611223] [INSPIRE].

[29] S. Schlamminger, K.Y. Choi, T.A. Wagner, J.H. Gundlach and E.G. Adelberger, Test of the equivalence principle using a rotating torsion balance, Phys. Rev. Lett. 100 (2008) 041101 [arXiv:0712.0607] [INSPIRE].

[30] K.-Y. Choi, J. Kim and C. Rott, Constraining dark matter-neutrino interactions with IceCube-170922A, Phys. Rev. D 99 (2019) 083018 [arXiv:1903.03302] [INSPIRE].

[31] N. Khan, Neutrino mass and the Higgs portal dark matter in the ESSFSM, Adv. High Energy Phys. 2018 (2018) 4809682 [arXiv: 1707.07300] [INSPIRE].

[32] A.Y. Smirnov, Neutrino and the dark side of the universe, ??? (2020).

[33] DAYA BAY collaboration, Measurement of the electron antineutrino oscillation with 1958 days of operation at Daya Bay, Phys. Rev. Lett. 121 (2018) 241805 [arXiv:1809.02261] [INSPIRE].

[34] RENO collaboration, Measurement of reactor antineutrino oscillation amplitude and frequency at RENO, Phys. Rev. Lett. 121 (2018) 201801 [arXiv:1806.00248] [INSPIRE].

[35] Double CHOOZ collaboration, Double CHOOZ $\theta_{13}$ measurement via total neutron capture detection, Nature Phys. 16 (2020) 558 [arXiv:1901.09445] [INSPIRE].

[36] T2K collaboration, Measurement of the charged-current electron (anti-)neutrino inclusive cross-sections at the T2K off-axis near detector ND280, JHEP 10 (2020) 114 [arXiv: 2002.11986] [INSPIRE].

[37] T2K collaboration, Constraint on the matter-antimatter symmetry-violating phase in neutrino oscillations, Nature $\mathbf{5 8 0}$ (2020) 339 [Erratum ibid. $\mathbf{5 8 3}$ (2020) E16] [arXiv: 1910.03887] [INSPIRE].

[38] NOvA collaboration, First measurement of neutrino oscillation parameters using neutrinos and antineutrinos by NOvA, Phys. Rev. Lett. 123 (2019) 151803 [arXiv:1906.04907] [INSPIRE].

[39] MINOS+ collaboration, Precision constraints for three-flavor neutrino oscillations from the full MINOS+ and MINOS dataset, Phys. Rev. Lett. 125 (2020) 131802 [arXiv:2006.15208] [INSPIRE].

[40] ICECuBE collaboration, Measurement of atmospheric tau neutrino appearance with IceCube DeepCore, Phys. Rev. D 99 (2019) 032007 [arXiv: 1901.05366] [InSPIRE].

[41] ANTARES collaboration, Measuring the atmospheric neutrino oscillation parameters and constraining the $3+1$ neutrino model with ten years of ANTARES data, JHEP 06 (2019) 113 [arXiv:1812.08650] [INSPIRE]. 